\title{
Direct numerical simulation of open-channel flow over smooth-to-rough and rough-to-smooth step changes
}

\author{
Amirreza Rouhi, Daniel Chung and Nicholas Hutchins \\ Department of Mechanical Engineering, University of Melbourne, Victoria 3010, Australia
}

(Received $\mathrm{xx}$; revised $\mathrm{xx}$; accepted $\mathrm{xx}$ )

Direct numerical simulations (DNSs) are reported for open-channel flow over streamwise-alternating patches of smooth and fully rough walls. The rough patch is a three-dimensional sinusoidal surface. Owing to the streamwise periodicity, the flow configuration consists of a step change from smooth to rough, and a step change from rough to smooth. The friction Reynolds number varies from 437 over the smooth patch to 704 over the rough patch. Through the fully resolved DNS dataset it is possible to explore many detailed aspects of this flow. Two aspects motivate this work. The first one is the equilibrium assumption that has been widely used both in experiments and computations. However, it is not clear where this assumption is valid. The detailed DNS data reveals a significant departure from equilibrium, in particular over the smooth patch. Over this patch the mean velocity is recovered up to the beginning of the log layer after a fetch of five times the channel height. However, over the rough patch same recovery level is reached after a fetch of two times the channel height. This conclusion is by assuming that an error up to $5 \%$ is acceptable and the log layer, classically, starts from 30 wall units above the wall. The second aspect is the reported internal boundary-layer (IBL) growth rates in the literature, which are inconsistent with each other. This is conjectured to be partly caused by the diverse IBL definitions. Five common definitions are applied on the same DNS dataset. The resulting IBL thicknesses are different by $100 \%$, and their apparent power-law exponents are different by $50 \%$. The IBL concept, as a layer within which the flow feels the surface underneath, is taken as the basis to search for the proper definition. The definition based on the logarithmic slope of the velocity profile, as proposed by Elliot (Trans. Am. Geophys. Union, vol. 39, 1958, pp. 1048-1054), yields better consistency with this concept based on turbulence characteristics.

Key words: Authors should not enter keywords on the manuscript, as these must be chosen by the author during the online submission process and will then be added during the typesetting process (see http://journals.cambridge.org/data/relatedlink/jfmkeywords.pdf for the full list)

\section{Introduction}

Changes in surface roughness occur in many fabricated or natural applications. Examples include the edges of forests, wind farms or the bio-fouled patches on a ship hull. Surface change may occur in the streamwise direction (Antonia \& Luxton 1971), spanwise direction (Anderson et al. 2015) or oblique to the flow direction. In more complex cases a 
(a)

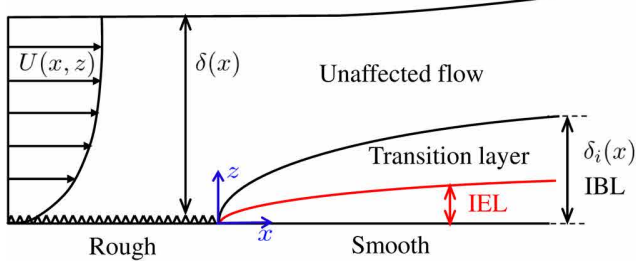

(b)

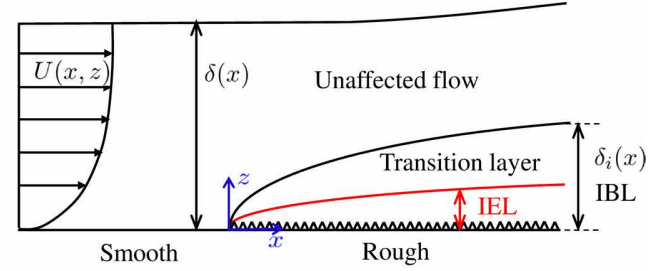

Figure 1: Schematic representation of the internal boundary-layer (IBL), internal equilibrium layer (IEL) and transition layer, adopted from Savelyev \& Taylor 2005, for a boundary layer over $(a)$ a rough-to-smooth step change, and $(b)$ a smooth-to-rough step change. $U(x, z)$ denotes the streamwise velocity averaged over time and spanwise direction, $\delta(x)$ and $\delta_{i}(x)$ are the boundary layer and IBL thicknesses, respectively.

combination of these surface changes may occur (Bou-Zeid et al. 2007; Yang \& Meneveau 2017). This study investigates the streamwise step change from a smooth surface to a rough surface, and vice-versa, collectively noted as streamwise-varying roughness.

Streamwise-varying roughness triggers various flow phenomena. Following the surface change the near-wall flow deviates from equilibrium. Depending on the surface change from smooth-to-rough or rough-to-smooth the surface drag increases or decreases. Consequently, the near-wall flow decelerates during smooth-to-rough surface change (Antonia \& Luxton 1971; Efros \& Krogstad 2011) and accelerates during roughto-smooth surface change (Antonia \& Luxton 1972; Mulhearn 1978). While the near-wall flow is affected by the new surface, the flow away from the wall still carries the history from the upstream surface (figure 1). The near-wall layer that is influenced by, but not necessarily in equilibrium with, the new surface is known as the internal boundarylayer, IBL (Kaimal \& Finnigan 1994; Brutsaert 1998; Savelyev \& Taylor 2005). The IBL thickness $\delta_{i}$ is the maximum height up to which the new surface effect is present, and separates the affected and unaffected regions. The lower part of the IBL that has reached equilibrium with the new surface is referred to as the internal equilibrium layer (IEL). The flow is still transitioning above the IEL and below $\delta_{i}$ (figure 1). The IEL is not the focus of this study and only the IBL is discussed. The IBL grows until it reaches the boundary-layer edge. At that point the flow recovers to a new equilibrium across the whole boundary layer. The recovery length depends on various factors including the surface properties, Reynolds number and the quantity of interest (Antonia \& Luxton 1971).

Streamwise-varying roughness has been investigated theoretically, numerically and experimentally (wind tunnel or field measurements). Here, only the numerical and wind tunnel experimental studies are reviewed, as are within the scope of this article. For interested readers some theoretical studies are Elliott (1958); Panofsky \& Townsend (1964); Calaf et al. (2010), and some field experiments are Miyake (1965); Bradley (1968); Munro \& Oke (1975).

The wind-tunnel experiments were conducted over a fabricated rough-to-smooth surface change, or vice-versa. The roughness geometries were composed of square bars (Antonia \& Luxton 1971, 1972; Efros \& Krogstad 2011; Jacobi \& McKeon 2011), grit roughness (Hanson \& Ganapathisubramani 2016), or mesh roughness (Carper \& Porté-Agel 2008; Chamorro \& Porté-Agel 2009; Hanson \& Ganapathisubramani 2016). The measuring devices varied depending on the parameter of interest. For the mean or r.m.s. velocity, studies used hot-wire anemometry (Antonia \& Luxton 1971, 1972; Cheng \& Castro 2002; Chamorro \& Porté-Agel 2009; Hanson \& Ganapathisubramani 
2016), Laser Doppler Anemometry (LDA) (Loureiro et al. 2010; Efros \& Krogstad 2011), or Particle-Image Velocimetry (PIV) (Carper \& Porté-Agel 2008; Jacobi \& McKeon 2011). To measure the wall shear-stress, due to the measurement difficulties over rough surfaces, the smooth surface following the rough-to-smooth step change was mostly emphasised (figure 1a). Studies have used the Preston tube (Antonia \& Luxton 1972; Hanson \& Ganapathisubramani 2016), Clauser fitting (Carper \& Porté-Agel 2008) or velocity gradient at the nearest measured point to the wall (Chamorro \& Porté-Agel 2009; Jacobi \& McKeon 2011).

The computational studies have mostly used Wall-Modelled Large Eddy Simulation (WMLES), or Reynolds-Averaged Navier-Stokes (RANS). For WMLES the near-wall region and hence the rough surface are modelled with a wall model. The commonly used wall model is the equilibrium logarithmic-law of the wall (Bou-Zeid et al. 2004; Silva-Lopes et al. 2015), and its extension to non-neutral flow using Monin-Obukhov similarity theory (Albertson \& Parlange 1999 a,b; Lin \& Glendening 2002; Stoll \& Porté-Agel 2006). The only fully resolved studies were the direct numerical simulations (DNSs) by Lee (2015) and Ismail et al. (2018). In both studies, rough surface was composed of square bars. However, Lee (2015) considered a smooth-to-rough step change in a boundary layer, while Ismail et al. (2018) considered a rough-to-smooth step change in a channel flow.

The computational studies differ from the wind-tunnel experiments in two aspects. First is the flow configuration, which is boundary layer in the experiments, while is typically full channel or open-channel flow in the computations. Second is $R e_{\tau}$, which is of the order $10^{3}$ in the experiments (Antonia \& Luxton 1971; Hanson \& Ganapathisubramani 2016), while is of the order $10^{5}-10^{6}$ in the WMLES studies (Miller \& Stoll 2013; Silva-Lopes et al. 2015) and is about 200-1000 in the DNS studies (Lee 2015; Ismail et al. 2018).

All the previous studies are invaluable in understanding the physics of the streamwisevarying roughness. Some aspects of this flow demand high-fidelity three-dimensional dataset. Two of these aspects that motivate this article are outlined below.

(i) Equilibrium assumption: In most of the experimental or numerical studies the measurements/calculations are performed from a certain height $z^{+}$(in wall-units) above the wall. Consequently, the missing near-wall region is modelled mostly with an equilibrium assumption. For instance, Carper \& Porté-Agel (2008) carried out a PIV study on the rough-to-smooth surface change at $R e_{\tau} \simeq 8800$. The first measured point was at $z^{+} \simeq 88$. Therefore, a Clauser fit was used to estimate the wall shear-stress. Antonia \& Luxton (1972) studied a rough-to-smooth step change at $R e_{\tau} \simeq 1700$. They used a Preston tube to measure the wall shear-stress. The tube diameter was $D^{+} \simeq 95$, implying that the equilibrium assumption was used up to $z^{+} \simeq 95$. They noticed $25 \%$ difference between the Preston tube wall shear-stress and the Clauser fit wall shear-stress. Hanson \& Ganapathisubramani (2016) studied a rough-to-smooth step change with a close $R e_{\tau}$ as Antonia \& Luxton (1972). They also used a Preston tube with a close $D^{+}$ as Antonia \& Luxton (1972). They obtained wall shear-stress close to Antonia \& Luxton (1972). Jacobi \& McKeon (2011) studied a perturbed boundary layer by a short rough patch at $R e_{\tau} \simeq 970-1200$. They measured the flow over the downstream smooth surface down to $z^{+} \simeq 3$. They conjectured that the viscous sublayer departed from equilibrium. Therefore, they instead used the wall shear stress of a canonical boundary layer for inner scaling. In the computational studies with WMLES Reynolds number is high. Therefore, equilibrium is assumed for a larger extent of the wall layer. For instance, in Saito \& Pullin (2014) at $R e_{\tau} \simeq 2 \times 10^{4}-2 \times 10^{6}$ the first grid point was at $z^{+} \simeq 410$. In Silva-Lopes et al. $(2015)$ at $R e_{\tau}=1.5 \times 10^{5}$ the first grid point was at $z^{+} \simeq 260$. Despite 
the extensive use of equilibrium assumption, it is not clear where this assumption is valid.

(ii) Internal boundary-layer: The IBL thickness $\delta_{i}$ has been quantified based on many definitions (table 1 of Savelyev \& Taylor 2005). $\delta_{i}$ and its growth rate (mostly described with a power law $\delta_{i} \propto x^{\alpha}$ ) appear to depend on $\delta_{i}$ definition. The studies that adopted the same definition obtained close $\alpha$. However, those that used different definitions, despite the similar flow conditions, obtained different $\alpha$. For instance, Cheng \& Castro (2002) and Lee (2015) studied a smooth-to-rough step change at $R e_{\tau}=2500$ and 180. They used the same definition (Pendergrass \& Arya 1984) and obtained close $\alpha$ (0.33, 0.22). Antonia \& Luxton (1971) and Win et al. (2010) studied a smooth-to-rough step change at $R e_{\tau}=2200$ and 2600. They used the same definition (Antonia \& Luxton $1971)$ and obtained close $\alpha(0.72,0.8)$. From these two pairs of studies, comparing Antonia \& Luxton (1971) with Cheng \& Castro (2002), the reported $\alpha$ differ by more than two times. However, both considered a smooth-to-rough step change at close $R e_{\tau}$. It is conjectured that $\delta_{i}$ definition is a major cause of discrepancy. A separate study that investigates this possibility is still missing.

This article aims to address the two above-mentioned aspects. For this purpose, DNSs of open-channel flow are performed with a bottom wall equally paved with smooth and rough patches. The presented DNSs differ from Lee (2015) and Ismail et al. (2018) in two aspects. First, the roughness here is a three-dimensional sinusoidal wall with the mean roughness height aligned with the smooth patch (figure 2). In Lee (2015) and Ismail et al. (2018) roughness is made of square bars with the mean height above the smooth patch. Second, here with the streamwise periodicity both rough-to-smooth and smooth-to-rough step changes are studied simultaneously. However, Lee (2015) only considered a smoothto-rough step change, and Ismail et al. (2018) only considered a rough-to-smooth step change. After describing the DNS setup $(\S 2)$, the results section starts with the domainlength study ( $\S 3.1)$. Then, the equilibrium assumption is investigated $(\S 3.2)$. Finally, the $\delta_{i}$ definitions are thoroughly studied to search for the most physically consistent choice $(\S 3.3)$.

\section{Direct Numerical Simulation}

The continuity and Navier-Stokes equations are solved in this study:

$$
\frac{\partial u_{i}}{\partial x_{i}}=0, \quad \frac{\partial u_{i}}{\partial t}+\frac{\partial u_{i} u_{j}}{\partial x_{j}}=G \delta_{i 1}-\frac{1}{\rho} \frac{\partial \widetilde{p}}{\partial x_{i}}+\nu \frac{\partial^{2} u_{i}}{\partial x_{j}^{2}}
$$

where $x_{1}, x_{2}$ and $x_{3}$ (or $x, y$ and $z$ ) are the streamwise, spanwise and wall-normal directions corresponding to the velocity components $u_{1}, u_{2}$ and $u_{3}$ (or $u, v$ and $w$ ), respectively. The pressure gradient $\partial p / \partial x_{i}$ has been decomposed into the constant volume and time averaged driving part $-\rho G$, and the periodic part $\partial \widetilde{p} / \partial x_{i}$.

Open-channel flow is the computational domain (figure 2). The bottom surface is equally divided between the smooth and rough patches. The smooth surface is aligned with the mean roughness height (figure $2 a$ ), and the $z$-coordinate origin is placed at the aligned height. The distance between the aligned height and the top boundary is denoted by $h$. Periodic boundary-conditions are imposed in the streamwise and spanwise directions. No-slip boundary condition is imposed on the bottom surface through an Immersed Boundary Method, IBM (Appendix A), and free-slip boundary condition is imposed on the top boundary. $G$ in $(2.1 b)$ is chosen such that the global Reynolds number $R e_{\tau_{o}} \equiv u_{\tau_{o}} h / \nu=590$, where $u_{\tau_{o}}$ is the friction velocity based on the total bottom wall drag, averaged over time and the entire bottom surface. Similar to a homogeneous channel 


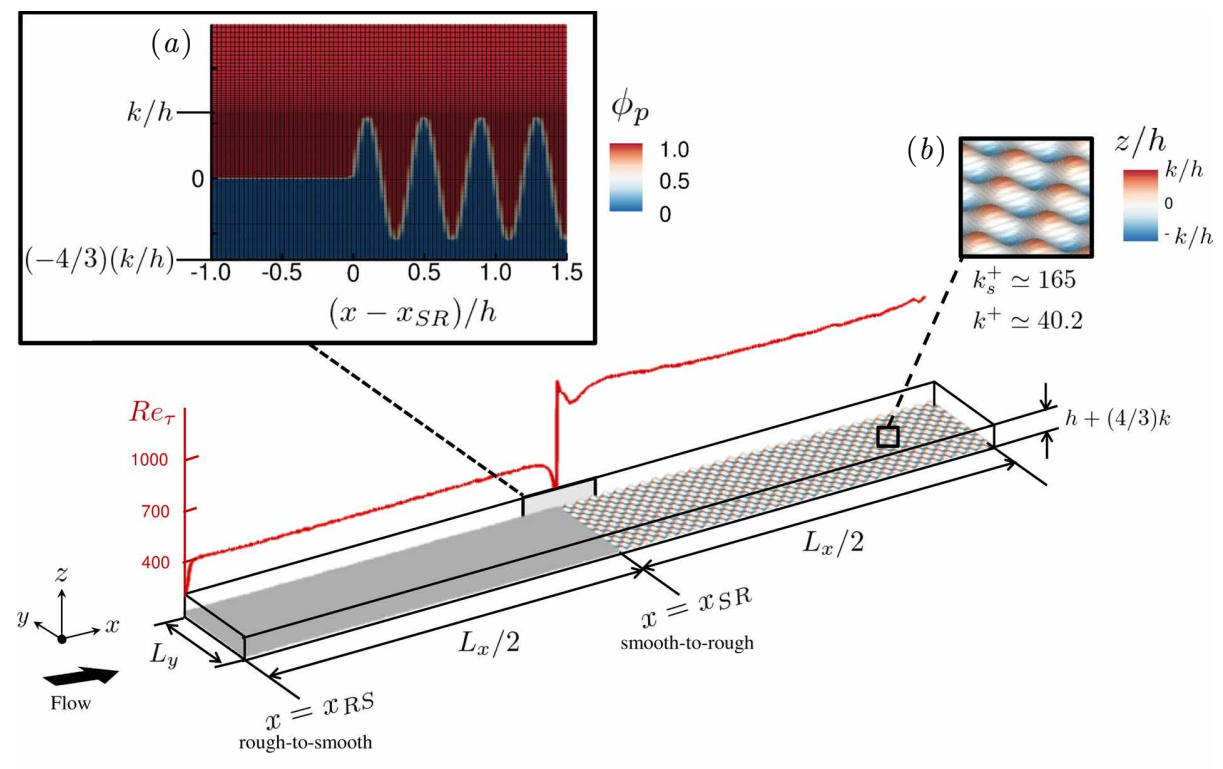

Figure 2: Computational domain equally divided between the smooth and rough patches. The bottom solid surface is identified with iso-surface of $\phi_{p}=0.5$. $\phi_{p}$ is the volume of fluid for the pressure cells (Appendix A). (a) side view of the domain, at the smooth-torough surface change overlaid by the grid. $(b)$ the roughness elements, coloured by $z / h$. The red curve is the friction Reynolds number $R e_{\tau} \equiv u_{\tau} h / \nu$ based on the local $u_{\tau}$.

flow, $G=u_{\tau_{o}}^{2} / h$. However, local $R e_{\tau} \equiv u_{\tau} h / \nu$ (based on local $u_{\tau}$ ) varies from about 700 over the rough patch to about 430 over the smooth patch (figure 2 ). The local $u_{\tau}$ accounts for both the viscous and form (pressure) drags, which is calculated by integrating the IBM force (appendix A). The bulk velocity is constant in each streamwise location. Therefore, the rough patch exerts a larger drag (larger $u_{\tau}$ ) than the smooth patch. In other words, $R e_{\tau}>590$ over the rough patch and $R e_{\tau}<590$ over the smooth patch.

The rough patch (figure $2 b$ ) is made of "egg-carton" roughness (Chan et al. 2015; Chung et al. 2015). The roughness surface $z_{r}$ is the following sinusoidal function:

$$
z_{r}=k \cos (2 \pi x / \lambda) \cos (2 \pi y / \lambda)
$$

where $k=0.056 h$ and $\lambda=7.1 k$ are the roughness height and wavelength, respectively. For the "egg carton" roughness, Chan et al. (2015) found that the mean roughness height is an appropriate choice for the virtual origin. Furthermore, Chan et al. (2015) and Chung et al. (2015) by fitting the data of this roughness geometry in the fully-rough regime, obtained the equivalent sand-grain roughness $k_{s} \simeq 4.1 k$. Therefore, with the current setup the flow falls into the fully rough regime over the rough patch, $k_{s}^{+} \simeq 165$. For further information on the geometrical characteristics of this type of roughness, the reader may refer to table 2 of Chan et al. (2015).

Equations $(2.1 a, b)$ are integrated in time using the fractional-step algorithm (Perot 1993). The time-marching scheme is the third-order Runge-Kutta (Spalart et al. 1991). Spatial discretisation is the fully conservative fourth-order symmetry-preserving scheme of Verstappen \& Veldman (2003). The reader may refer to Appendix A for details of the numerical scheme, IBM, and verification against a body-conforming grid solver.

Three cases are considered whose domain sizes and grid resolutions are listed in table 1 . 


$\begin{array}{cccccccc}\text { Case } & L_{x} / h & N_{x} \times N_{y} \times N_{z} & \Delta_{x_{s}}^{+}, \Delta_{x_{r}}^{+} & \Delta_{y_{s}}^{+}, \Delta_{y_{r}}^{+} & \left.\Delta_{z_{s}}^{+}\right|_{0},\left.\Delta_{z_{r}}^{+}\right|_{0} & \lambda / \Delta_{x} & \lambda / \Delta_{y} \\ 6 \mathrm{~h} & 6.06 & 384 \times 384 \times 400 & 6.9,11.9 & 3.6,6.3 & 0.3,0.5 & 25.2 & 48.0 \\ 12 \mathrm{~h} & 12.03 & 768 \times 384 \times 400 & 6.7,11.5 & 3.5,6.1 & 0.2,0.4 & 25.4 & 48.0 \\ 24 \mathrm{~h} & 23.96 & 1536 \times 384 \times 400 & 6.7,11.9 & 3.5,6.3 & 0.2,0.5 & 25.5 & 48.0\end{array}$

Table 1: Domain size and grid resolution information. For all cases $R e_{\tau_{o}}=590$ (based on the global $u_{\tau_{o}}$ and $h$ ) and $L_{y} / h=3.1808 . \Delta_{x_{s}}^{+}, \Delta_{y_{s}}^{+}$and $\left.\Delta_{z_{s}}^{+}\right|_{0}$ are scaled by the $u_{\tau}$ at a fetch of $2 h$ over the smooth patch. $\Delta_{x_{r}}^{+}, \Delta_{y_{r}}^{+}$and $\left.\Delta_{z_{r}}^{+}\right|_{0}$ are scaled by the $u_{\tau}$ at a fetch of $2 h$ over the rough patch. $\left.\Delta_{z_{s}}^{+}\right|_{0},\left.\Delta_{z_{r}}^{+}\right|_{0}$ are the near wall $\Delta z^{+}$at $z=0 . \lambda / \Delta_{x}$ and $\lambda / \Delta_{y}$ indicate the number of grid points per roughness wavelength in the streamwise and spanwise directions, respectively.

For these cases all the input parameters are the same except the domain lengths $(6 h, 12 h$ and 24h). Uniform grid spacing is used in the streamwise and spanwise directions. For the wall-normal grid, a uniform distribution with $\Delta_{z} u_{\tau_{o}} / \nu=0.35$ is generated up to the roughness crest, and then is stretched up to the top boundary in a tangent-hyperbolic mapping (figure $2 a$ ). The grid sizes are normalised by the local $u_{\tau}$ at a fetch of $2 h$ over the smooth patch $\left(\Delta x_{s}^{+}, \Delta y_{s}^{+},\left.\Delta_{z_{s}}^{+}\right|_{0}\right)$, and at a fetch of $2 h$ over the rough patch $\left(\Delta x_{r}^{+}, \Delta y_{r}^{+},\left.\Delta_{z_{r}}^{+}\right|_{0}\right)$. The reason for measuring the resolution at a distance of $2 h$ is the small variation in the local $u_{\tau}$ (less than $6 \%$ ) beyond a fetch of $2 h$. The choice of the resolutions in table 1 are from various verification studies (Appendix A).

To ease the discussion, the $x$-coordinate at the rough-to-smooth step change is $x_{R S}$, and at the smooth-to-rough step change is $x_{S R}$ (figure 2). The statistics over the smooth patch are averaged over time and spanwise directions. Over the rough patch first the statistics are averaged over time and spanwise directions, considering only the in-fluid cells. Then are streamwise averaged from a distance of $\lambda / 2$ upstream to $\lambda / 2$ downstream. For locations with distances less than $\lambda / 2$ to $x_{S R}$ or $x_{R S}$, the averaging window is constrained by the distance to $x_{S R}$ or $x_{R S}$. Throughout this article $U, W$ and $P$ denote the streamwise and wall-normal mean velocities, and mean pressure, respectively. $u_{r m s}, v_{r m s}$ and $w_{r m s}$ are the r.m.s. of streamwise, spanwise and wall-normal fluctuating velocities, respectively. All these statistics are averaged following the described procedure. Also $\langle$.$\rangle by default$ denotes the same averaging procedure (i.e. $\langle u\rangle=U$ ), unless it appears with a subscript (i.e. $\langle u\rangle_{t}$ is time averaged $u$ ). All the parameters in plus units $(.)^{+}$are normalised by the local $u_{\tau}$ and $\nu$ (where $u_{\tau}$ is averaged following the averaging procedure described).

\section{Results}

The results are presented in three sections. In $\S 3.1$ the parameters of interest are shown insensitive to the domain length and streamwise periodicity. In $\S 3.2$ equilibrium assumption and its range of validity is studied. Finally, in $\S 3.3 \delta_{i}$ definitions are studied to find the most physical choice.

\subsection{Domain-length effect}

In the streamwise-varying roughness, flow recovery is slow ( $\S 3.2$ ). Full recovery is reached after a fetch of $64 h$ (Saito \& Pullin 2014). Consequently, the previous wind tunnel experiments (Antonia \& Luxton 1971; Hanson \& Ganapathisubramani 2016) or DNS studies (Lee 2015; Ismail et al. 2018) do not reach full recovery due to development lengths that are less than $20 \delta$ (or 20h). However, full recovery is not the focus of this study. 
(a)
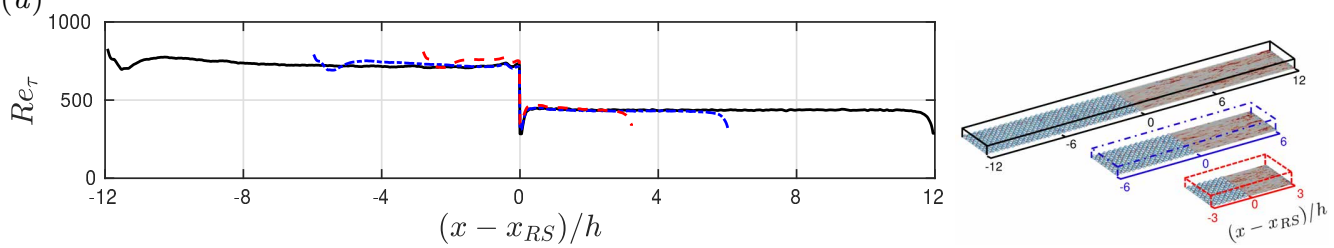

(b)
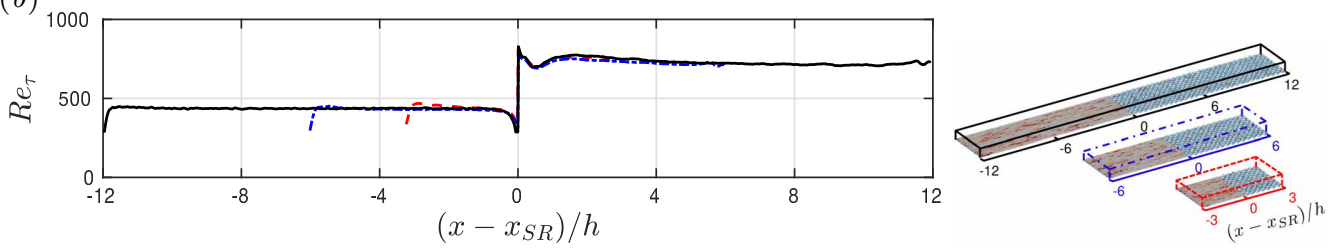

Figure 3: Comparison of the local $R e_{\tau}$ between case $6 \mathrm{~h}(---), 12 \mathrm{~h}(-\cdot-)$ and $24 \mathrm{~h}($ ), the cases on the right. Comparison over $(a)$ the smooth patch and $(b)$ the rough patch. The $x$-origin is placed at $(a) x_{R S}$ and $(b) x_{S R}$.

The focus here is on the flow within the IBL in the near-field of the surface transition. Given the finite patch length and streamwise periodicity, the unrecovered flow prior to the surface change will in general influence the downstream flow. However, Bou-Zeid et al. (2005) also simulated step changes in a periodic open-channel setup to replicate the measurements of Bradley (1968). They argued that the near wall flow (within the IBL) was insensitive to domain periodicity. Here, this insensitivity is verified by comparing the three domain lengths of table 1. Additionally, in Appendix B case $12 \mathrm{~h}$ is compared with a non-periodic rough-to-smooth case, where fully recovered flow over rough wall is imposed to the inlet, at the beginning of the smooth patch.

The patch length effect on $R e_{\tau}$ is studied over the smooth patch (figure $3 a$ ) and the rough patch (figure $3 b$ ). The origin has been placed at the beginning of the corresponding patch, to better isolate the domain-length effect. Except the shortest domain length (case $6 \mathrm{~h}$ ), the two longer cases yield almost identical $R e_{\tau}$ over both the smooth patch (figure $3 a$ ) and the rough patch (figure $3 b$ ). Even the maximum difference between case $6 \mathrm{~h}$ and the two longer cases is only $6.7 \%\left(\right.$ near $\left.x_{R S}\right)$.

The patch-length effect on $U^{+}$and $u_{r m s}^{+}$is studied over the smooth patch (figure 4) and the rough patch (figure 5). The IBL thickness $\delta_{E}\left(--0_{--}\right)$(defined by Elliott 1958 and discussed in $\S 3.3$ ), has been overlaid on the contour lines. Within the IBL, cases $12 \mathrm{~h}$ and $24 \mathrm{~h}$ yield almost identical $U^{+}$and $u_{r m s}^{+}$. This is better demonstrated by comparing the $U^{+}$and $u_{r m s}^{+}$profiles up to a fetch of $2.5 h$ over the smooth patch (figures $4 b, d$ ) and over the rough patch (figures $5 b, d$ ). Within the IBL, the maximum difference between case $12 \mathrm{~h}$ and $24 \mathrm{~h}$ in the $U^{+}$profiles is $1 \%$ over both the smooth patch (figure $4 b$ ) and the rough patch (figures $5 b$ ). Within the IBL, the maximum difference in the $u_{r m s}^{+}$profiles is $4 \%$ over the smooth patch (figure $4 d$ ) and $1 \%$ over the rough patch (figure $5 d$ ). As a further support for the small dependence on the domain length, the IBL thicknesses are compared in figure 6 . The maximum difference between case $12 \mathrm{~h}$ and $24 \mathrm{~h}$ is $5 \%$ over the smooth patch (figure $6 a$ ), and $3 \%$ over the rough patch (figure $6 b$ ). Similar to the findings here, in Appendix B negligible difference within the IBL is seen between case $12 \mathrm{~h}$ and the non-periodic case; the difference is less than $1 \%$ in $U^{+}$, and $4 \%$ in $u_{r m s}^{+}$and $R e_{\tau}$.

The identical statistics below $\delta_{i}$ and the differences above $\delta_{i}$ is justifiable through the IBL concept: a layer that is influenced by the surface underneath. Below $\delta_{i}$ the flow 


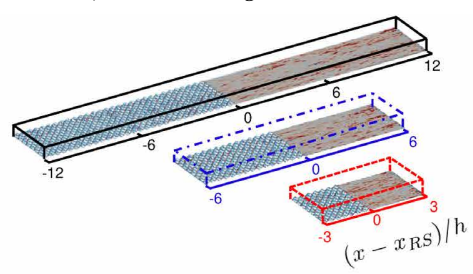

(a) $U^{+}$

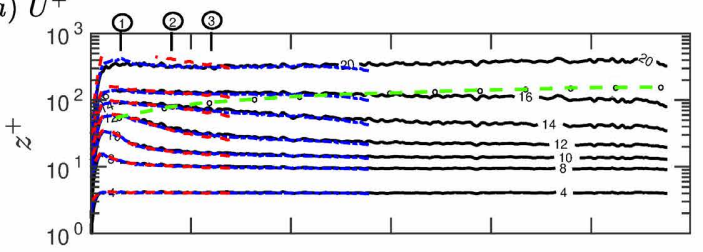

(c) $u_{r m s}^{+}$

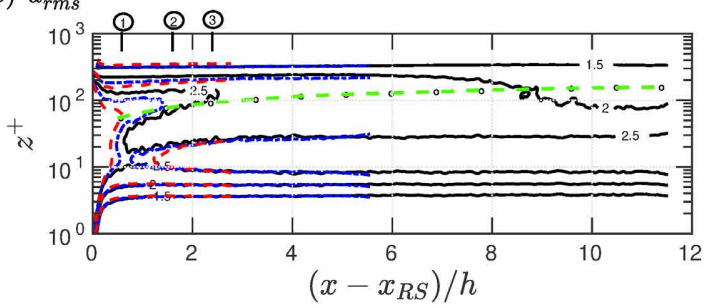

(b)

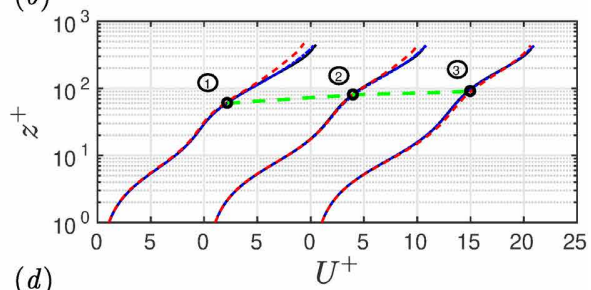

$(d)$

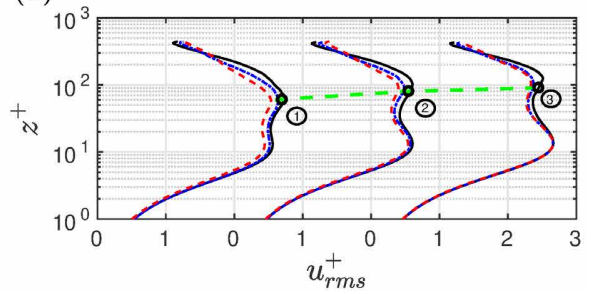

Figure 4: Contour lines of $(a) U^{+}$and $(c) u_{r m s}^{+}$for the three domain lengths over the smooth patch. The $x$-origin is placed at $x_{R S}$ (consider the top domains). Profiles of $(b)$ $U^{+}$and $(d) u_{r m s}^{+}$at several $\left(x-x_{R S}\right)$ : (1) $0.5 h$, (2) $1.5 h$ and (3) $2.5 h$. Legends are consistent with figure 3. Quantities in plus units are scaled by the local $u_{\tau}$ and $\nu$. The IBL thickness, defined by Elliott (1958), is overlaid on the contour lines and profiles (-- o-- ).

ignores its history from the upstream surfaces. Therefore, it has minimal dependence on the patch length. Above $\delta_{i}$, however, the flow carries its history from upstream surfaces. Therefore, it depends on the patch length. This section and Appendix B show that with domain lengths of at least $12 h$ ( $6 h$ for each patch), the flow inside the IBL remains insensitive to the patch length and streamwise periodicity. The results reported in the rest of this paper are from the longest case (case $24 \mathrm{~h}$ ).

\subsection{Equilibrium assumption}

In this section validity of the equilibrium assumption is examined. First, the overall flow behaviour is described (figure 7 ). The quantities are scaled by the bulk velocity $U_{b} \simeq 12.78 u_{\tau_{o}}$ and channel height $h$. For ease of discussion, each patch is divided into two zones: S1 and S2 over the smooth patch, and R1 and R2 over the rough patch. Zones S1 and R1 cover up to a fetch of $2 h$, where the flow variations are rapid. Zones S2 and $\mathrm{R} 2$ cover the remaining portions, where the flow variations are more gradual. As a measure of the flow acceleration or deceleration, $\left(h / U_{b}\right)(\partial U / \partial x)$ is plotted in figure $7(b)$. In figure $7(c)$ the pressure gradient $\partial P / \partial x$ includes both the driving part $(-\rho G)$ and the periodic part $(\partial \widetilde{P} / \partial x)$. During the step change the periodic $\partial \widetilde{P} / \partial x$ becomes an order of magnitude larger than the driving $-\rho G$. In other words, $h G / U_{b}^{2} \simeq 6 \times 10^{-3}$ which is not visible with the colour range in figure $7(c)$.

In figure $7(b)$ following the rough-to-smooth step change the near-wall flow accelerates, while away from the wall the flow decelerates. This is because $d U_{b} / d x=\int_{0}^{h}(\partial U / \partial x) d z=$ 

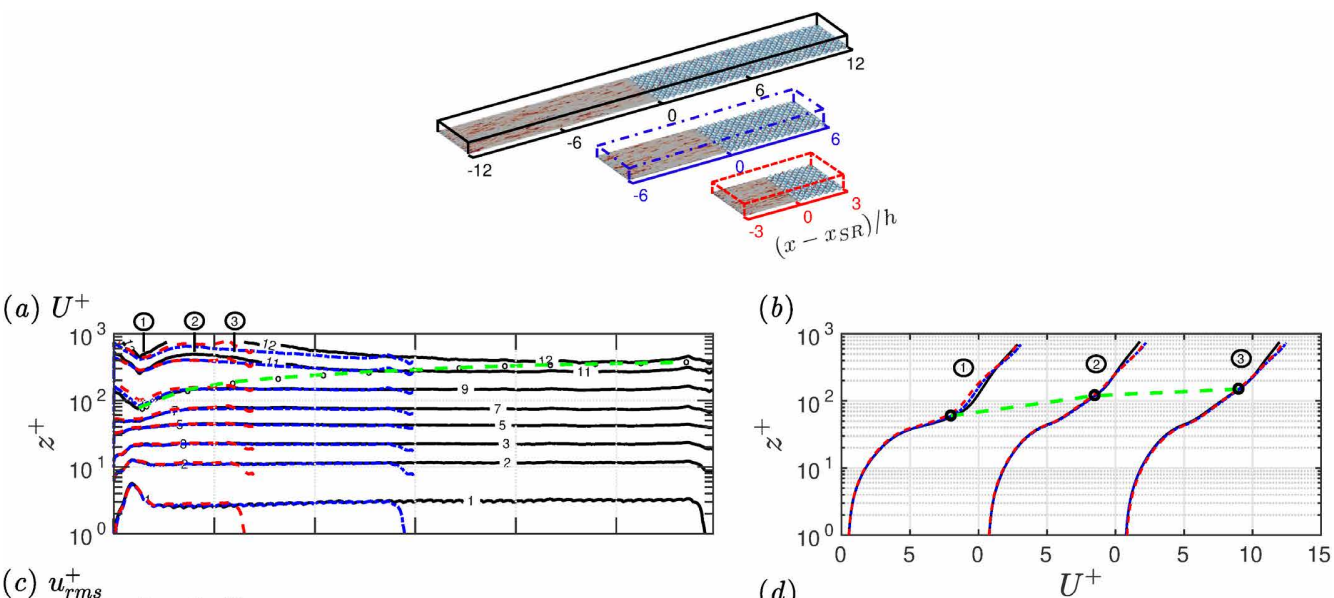

(c) $u_{r m s}^{+}$
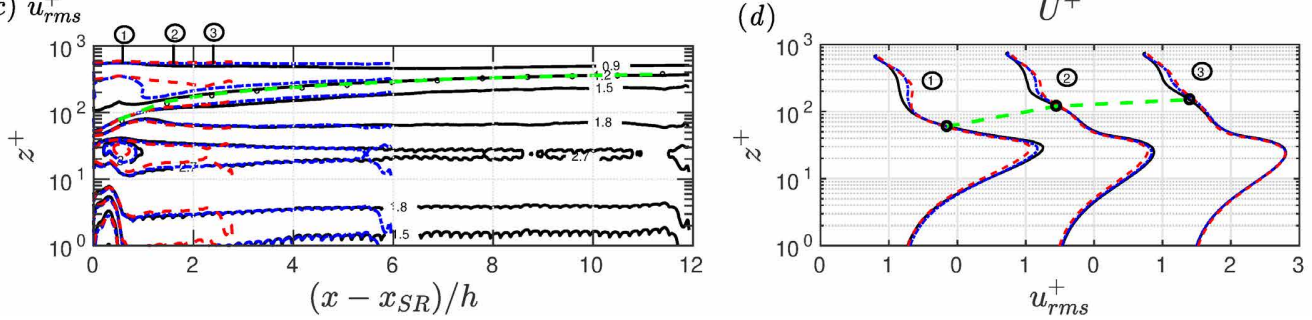

Figure 5: Contour lines of $(a) U^{+}$and $(c) u_{r m s}^{+}$for the three domain lengths over the rough patch. The $x$-origin is placed at $x_{S R}$ (consider the top domains). Profiles of $(b) U^{+}$ and $(d) u_{r m s}^{+}$at several $\left(x-x_{S R}\right)$ : (1) $0.5 h$, (2) $1.5 h$ and (3) $2.5 h$. Legends are consistent with figure 3. Quantities in plus units are normalised by the local $u_{\tau}$ and $\nu$. The IBL thickness, defined by Elliott (1958), is overlaid on the contour lines and profiles (-- o - - ).
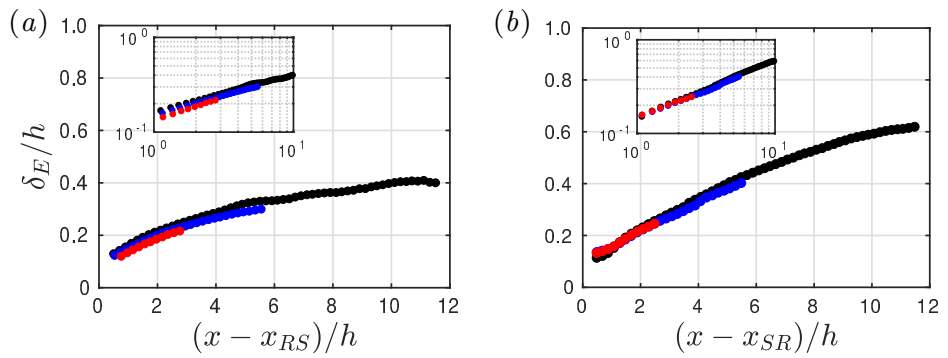

Figure 6: IBL thickness defined by Elliott (1958) $\left(\delta_{E}\right.$, discussed in $\left.\S 3.3\right)$ over $(a)$ the smooth patch and $(b)$ the rough patch. Case $6 \mathrm{~h}(\bullet), 12 \mathrm{~h}(\bullet)$ and $24 \mathrm{~h}(\bullet)$. The insets are the same plots in log-log scale.

0 , and $\partial U / \partial x>0$ near the wall must be accompanied by $\partial U / \partial x<0$ away from the wall. Simultaneously, the flow is exposed to an adverse pressure-gradient $(\partial P / \partial x>0)$, which becomes strong at the beginning of zone S1 (figure $7 c$ ). Following the smoothto-rough step change, the acceleration/deceleration mechanism is reversed: the nearwall flow decelerates while the outer one accelerates, and the flow is exposed to a favourable pressure-gradient. In figure $7(d)$ immediately downstream of the rough-tosmooth step change (zone S1), the wall-normal flow direction is downward $(W<0)$, while immediately downstream of the smooth-to-rough step change (zone R1), the wall- 
(a) $U / U_{b}$

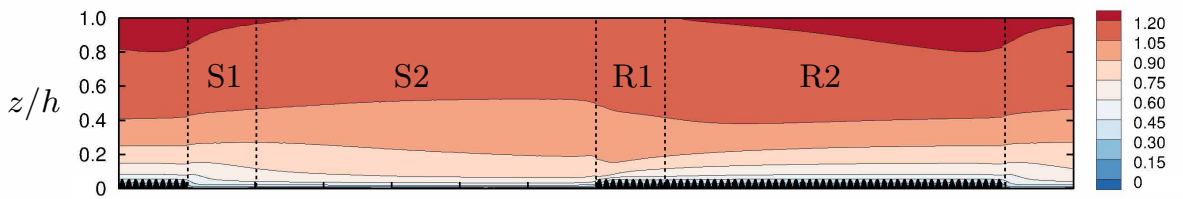

(b) $\left(h / U_{b}\right)(\partial U / \partial x)$

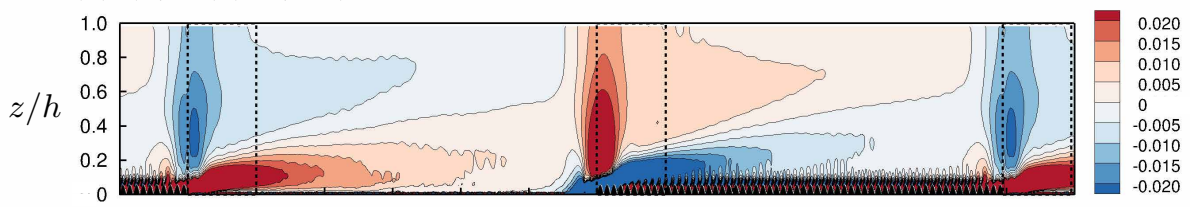

(c) $\left[h /\left(\rho U_{b}^{2}\right)\right](\partial P / \partial x)$

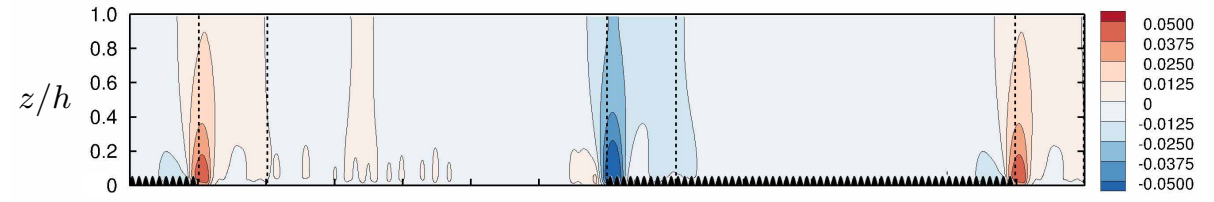

(d) $W / U_{b}$

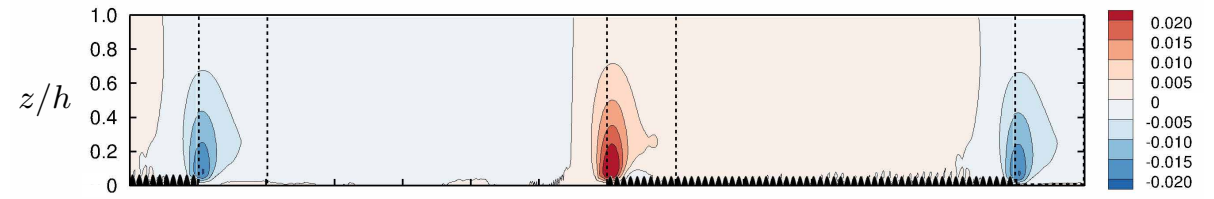

(e) $u_{r m s} / U_{b}$

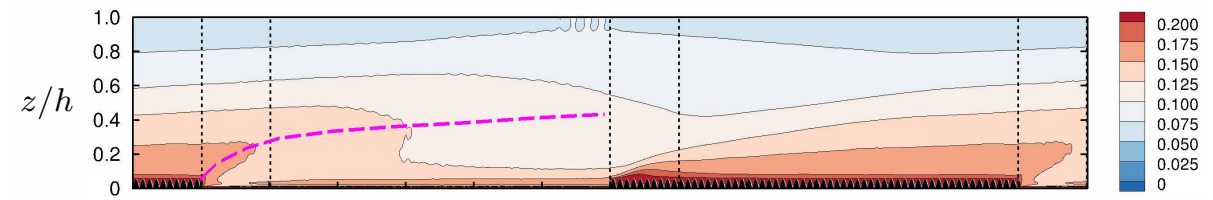

(f) $u_{r m s} / U_{b}$

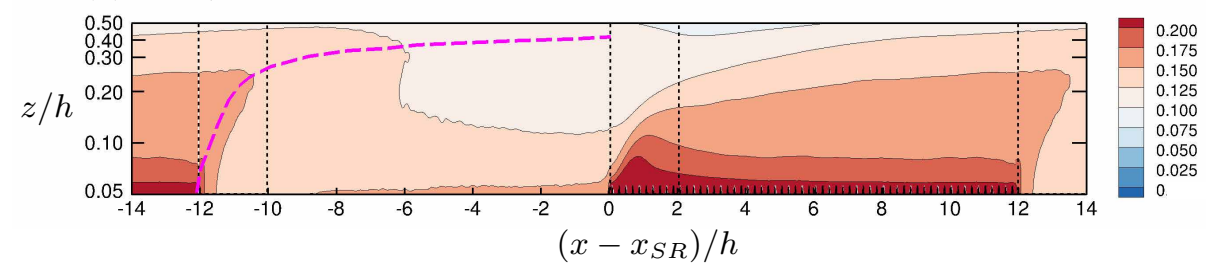

Figure 7: Variations of $(a) U,(b) \partial U / \partial x,(c) \partial P / \partial x,(d) W$, and $(e, f) u_{r m s}$, scaled by the bulk velocity $U_{b}$ and $h$. The regions over the smooth patch $(\mathrm{S} 1+\mathrm{S} 2)$ and rough patch $(\mathrm{R} 1+\mathrm{R} 2)$ are separated into zones $\mathrm{S} 1$ and $\mathrm{R} 1$ that cover a fetch of $2 h$, and zones $\mathrm{S} 2$ and $\mathrm{R} 2$ that cover the remaining portions. The fields are overlaid by the spanwise projection of the roughness, in black colour. In $(c)$ the total pressure-gradient $\partial P / \partial x$ includes the driving $-\rho G$ and periodic $\partial \widetilde{P} / \partial x$ parts. In $(f)$ the $z$-axis is in log scale to highlight the near wall region. The outer peak of $u_{r m s}$ over the smooth patch is marked with (--- ). 
normal flow direction is upward $(W>0)$. This behaviour is justifiable through the continuity equation, $\partial U / \partial x+\partial W / \partial z=0$. In zone $\mathrm{S} 1, \partial U / \partial x>0$ near the wall requires $\partial W / \partial z<0$, and since $W=0$ at the wall, $W$ must be negative near the wall. The same analysis justifies positive $W$ in zone R1.

Some interesting phenomena are seen in the $u_{r m s}$ field (figure $7 e, f$ ). Immediately downstream of the rough-to-smooth step change, there is a locally high $u_{r m s}$ region (at $x-x_{S R} \simeq-12 h$ and $\left.z \simeq 0.05 h\right)$. Along the smooth patch $u_{r m s}$ near the wall $(z \lesssim 0.5 h)$ is decreased, while away from the wall $(z>0.5 h)$ it preserves its intensity. This leads to formation of an outer peak in the $u_{r m s}$ field (marked with the dashed magenta curve). Immediately downstream of the smooth-to-rough step change (at $x-x_{S R} \simeq 0$ ) there is a sudden rise in $u_{r m s}$. Along the rough patch the high intensity $u_{r m s}$ around the roughness elements gradually propagates to higher $z$ distances. These phenomena are further investigated next.

\subsubsection{Rough-to-smooth step change}

The profiles of $U$ and $u_{r m s}$ up to a fetch of $2 h$ over the smooth patch are shown in figure 8 . The profiles are scaled by $U_{b}$ and $h$ in figure $8(a, b)$, local $u_{\tau}$ and $\nu$ in figure $8(c, d)$, and $u_{\tau_{o}}$ and $\nu$ in figure $8(e, f)$. Over the smooth patch $R e_{\tau}$ converges to the asymptotic value of 437 . Therefore, to measure the flow distance to equilibrium, a separate simulation of fully developed smooth open-channel flow at $R e_{\tau}=437$ was conducted $\left(L_{x} / h \times L_{y} / h=2 \pi \times \pi, \Delta_{x}^{+} \times \Delta_{y}^{+} \simeq 10.7 \times 5.4\right)$.

In figure $8(c)$ the $U^{+}$profiles are substantially departed from equilibrium. The departure even propagates down to the buffer and viscous sublayer regions $\left(z^{+} \lesssim 30\right)$. Due to the thinner buffer layer, a downshift appears in the $U^{+}$profiles. Similar downshift is seen in the Adverse Pressure Gradient (APG) boundary layers (Nickels 2004). When the APG strength $P_{x}^{+}, \nu /\left(\rho u_{\tau}^{3}\right)(\partial P / \partial x)$, goes beyond 0.005 , it breaks the linear viscous sublayer. Here, from the beginning of the smooth patch up to a fetch of $2 h, P_{x}^{+}$varies from 0.022 to 0.001 (not shown). Therefore, it is possible that APG is causing the downshift in the $U^{+}$profiles. This possibility was examined by reconstructing the $U^{+}$profiles using the obtained $P_{x}^{+}$from DNS, substituted in Nickel's formulation for the viscous sub-layer $\left(U^{+}=z^{+}+1 / 2 P_{x}^{+} z^{+^{2}}+\right.$ h.o.t) and the $\log$ layer (equation 3.1 in Nickels 2004). At each $x$-location $P_{x}^{+}$is constant for $z^{+} \leq 100$. The reconstructed profiles had a much sallower downshift than what is seen in figure $8(c)$. Therefore, the downshift is not merely caused by APG.

One can also see that there is a change in the logarithmic slope of the $U^{+}$profiles across the channel. This is better demonstrated in figure $9(a)$ showing the $U^{+}$profile at $\left(x-x_{R S}\right)=2 h$. To detect the slope change, the slope curve $\partial U^{+} / \partial \ln \left(z^{+}\right)$at $(x-$

$\left.x_{R S}\right)=2 h(-)$ is compared with the equilibrium counterpart (+) in figure $9(b)$. For equilibrium open-channel flow $\partial U^{+} / \partial \ln \left(z^{+}\right)$yields almost a plateau for $40 \lesssim z^{+} \lesssim 300$, indicating that the logarithmic region dominates the wake in this range. This is clear when compared to a canonical boundary layer (o) at similarly matched $R e_{\tau} \simeq 445$ (Jiménez et al. 2010), which yields a narrow logarithmic region but a strong outer wake. During the rough-to-smooth step change (— ) the slope curve yields a local minimum $(\bullet)$ and a local maximum $(\bullet)$ at $z^{+} \simeq 40$ and $z^{+} \simeq 200$, indicating the inner and outer logarithmic slopes, respectively. The inner slope reflects the influence of the new smooth surface, while the outer slope owing to the weak channel wake predominantly reflects the flow history from the upstream rough surface. The new surface effect can also be seen in figure $8(e)$ comparing the $U$ profiles with their most upstream counterpart (the green curve). The extent up to which each profile is departed from the green curve (which also appears as the inner logarithmic slope), is the result of the new surface underneath. 

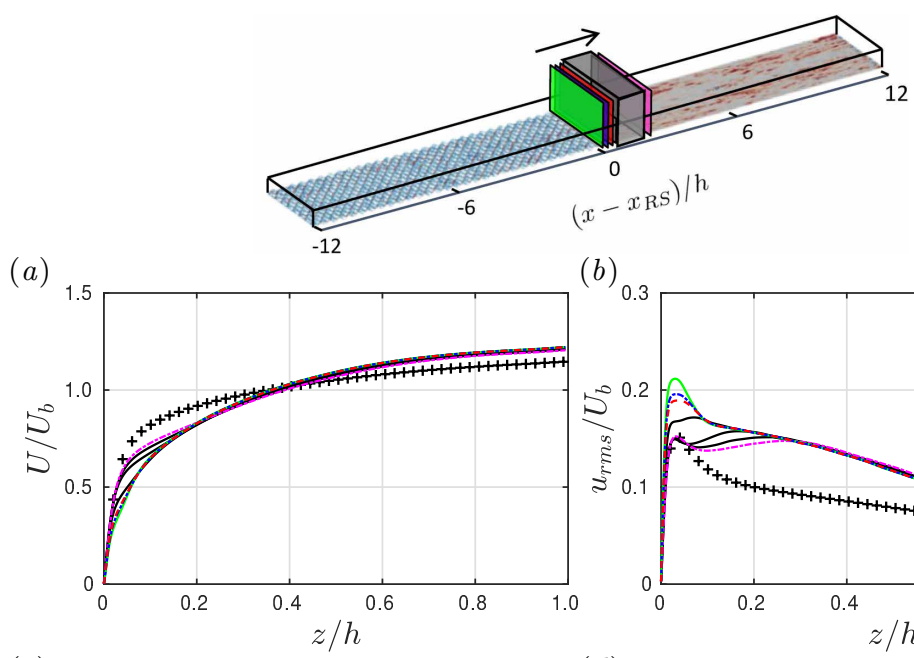

(c)
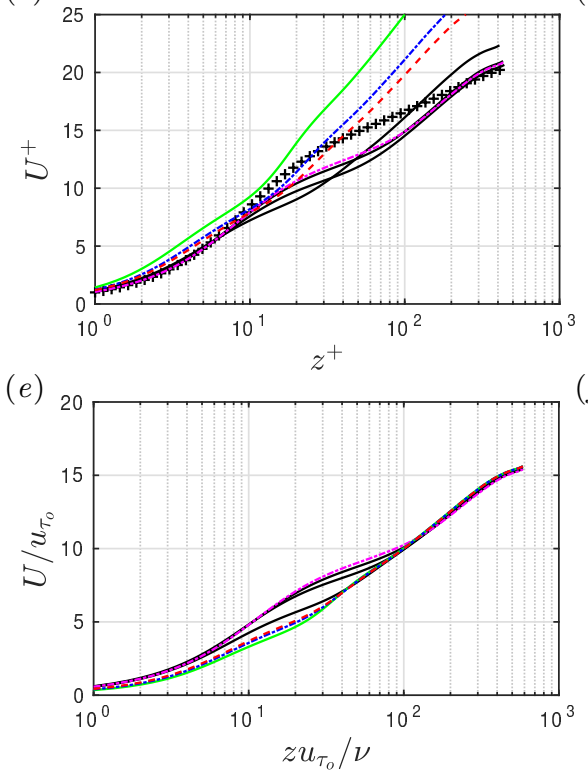

(b)

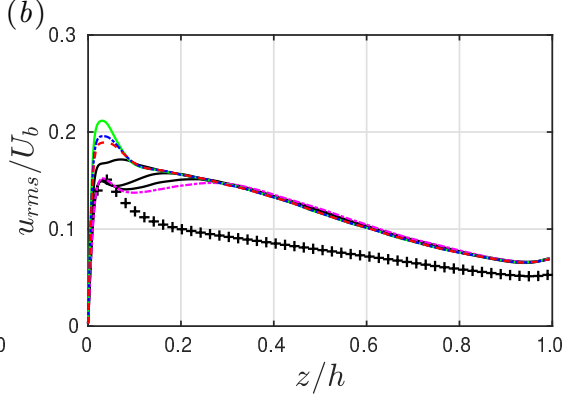

$(d)$
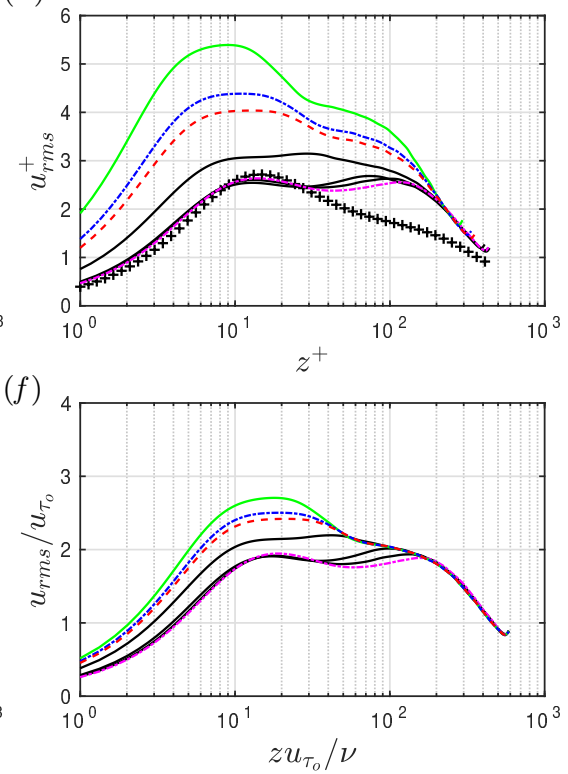

Figure 8: Profiles of $(a, c, e) U$, and $(b, d, f) u_{r m s}$ up to a fetch of $2 h$ over the smooth patch, zone S1. profiles are normalised by $(a, b) U_{b}, h,(c, d)$ local $u_{\tau}$ and $\nu$ and $(e, f) u_{\tau_{o}}$ and $\nu$. The black curves are equally spaced in the range $0.2 h \leq\left(x-x_{R S}\right) \leq 1.8 h$ (the shadowed box at the top). $\left(x-x_{R S}\right)=0.05 h(-), 0.08 h(-\cdot-), 0.1 h(---)$ and $2 h$ (--- ); DNS of fully developed smooth open-channel flow at $R e_{\tau}=437(+)$.

In figure $8(b, d, f)$ the $u_{r m s}$ profile at the very beginning of the smooth patch (the green curve at $x-x_{R S}=0.05 h$ ) yields a large inner peak (at $z^{+} \simeq 9$ ). This peak corresponds to the high near-wall $u_{r m s}$ appearing immediately downstream of the roughto-smooth step change, discussed earlier in figure $7(e, f)$. It is the remnant of the turbulent fluctuations emanated from the upstream rough patch. This peak is different than the inner peak formed further downstream due to the buffer layer formation (the magenta dashed-dotted curve at $z^{+} \simeq 14$ ). This is better shown in figure 10, comparing the $u_{r m s}$ profiles immediately upstream of the rough-to-smooth step change $\left(x-x_{R S}=-0.05 h\right)$ 

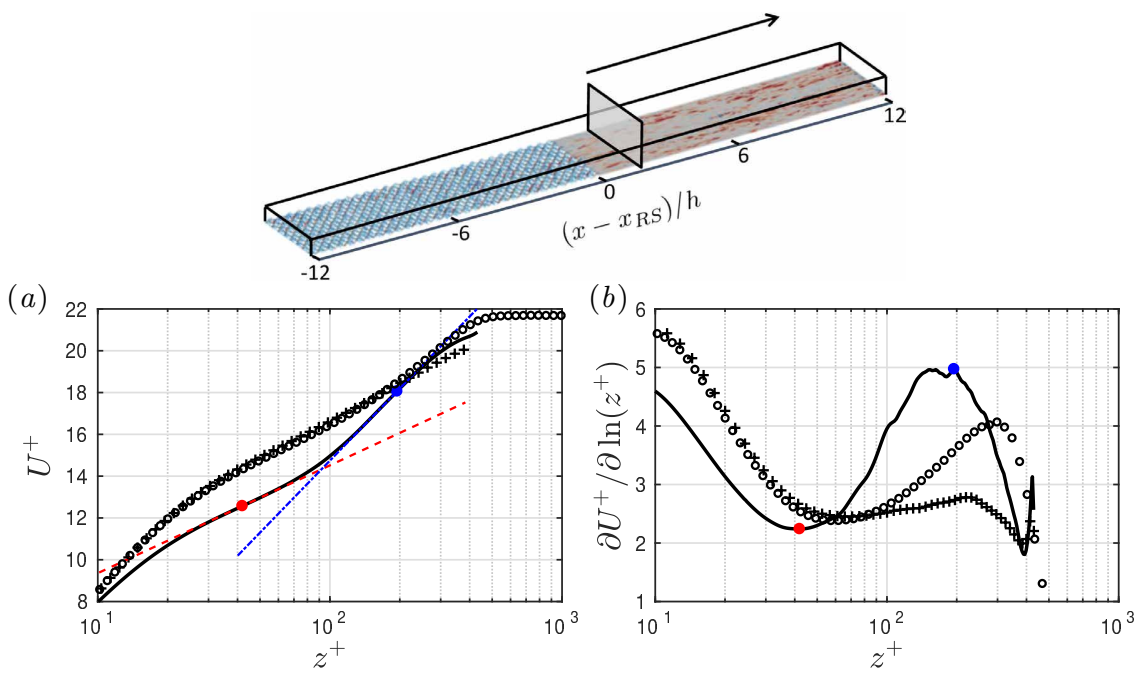

Figure 9: Profiles of $(a) U^{+}$and $(b)$ its logarithmic slope $\partial U^{+} / \partial \ln \left(z^{+}\right)$for case $24 \mathrm{~h}$ at $\left(x-x_{R S}\right)=2 h(-)$, indicated in the top domain. The inner and outer logarithmic slopes are identified through the extrema of $\partial U^{+} / \partial \ln \left(z^{+}\right)(\bullet, \bullet)$. The fitting lines $(---)$ and (---) have the same slopes as the extrema. Fully developed open-channel flow at $R e_{\tau}=437(+)$. Canonical boundary layer at $R e_{\tau} \simeq 445$ (०) by Jiménez et al. (2010).

with the profiles immediately downstream of the step change $\left(x-x_{R S}=0.05 h\right)$ and at a fetch of $2 h\left(x-x_{R S}=2 h\right)$. To make the profiles comparable, they are scaled by $U_{b}$. As is seen the inner peak immediately downstream of the step change (the green solid curve) is a weakened remnant of the inner peak immediately upstream of the step change (the black dotted curve). Further downstream at $\left(x-x_{R S}\right)=2 h$ (the magenta dashed-dotted curve), two different peaks appear which are identified by arrows. The inner peak (the upward arrow) is due to the buffer layer formation, and the outer one (the downward arrow) is due to the surface change. The magenta curve matches the most upstream profile (the green solid curve) beyond the outer peak location. Along the smooth patch the outer peak moves to a higher $z$ (figure $8 f$ ), locating the maximum height up to which $u_{r m s}$ is influenced by the surface underneath. This outer peak is marked with magenta dashed curve in figure $7(e, f)$.

The profiles of $U$ and $u_{r m s}$ in the remaining portion of the smooth patch, zone S2, are shown in figure 11. In this figure the $x$-distance between the first and the last profile is four times larger than the one in figure 8. However, the profiles variation is much slower. In other words, the recovery for the initial $2 h$ fetch length is much faster than the remaining portion. By the end of the smooth patch, the flow is still not fully recovered. The effect of the upstream rough patch still persists in the $u_{r m s}$ (figure $11 b, d, f$ ), as well as the $U$ profile (figure $11 a, c, e$ ).

Recovery of $U^{+}$over the smooth patch is compared with the DNS of rough-to-smooth by Ismail et al. (2018) in figure 12 and table 3. The configuration of Ismail et al. (2018) differs from the current case (case $24 \mathrm{~h}$ ) in several aspects (table 2). These aspects include: $R e_{\tau}$, roughness shape, roughness size and roughness origin. Considering figure 12, initially at $\left(x-x_{R S}\right)=0.8 h$ (figure $12 a$ ) the $U^{+}$profile of Ismail et al. (2018) yields a larger departure from equilibrium. Nevertheless, after a fetch of $\left(x-x_{R S}\right)=4.1 h$ (figure $12 c$ ) the $U^{+}$profile of both datasets reach the same recovery level. This is better quantified in table 3 , which reports the $z^{+}$up to which $U^{+}$differs from the fully developed profile 


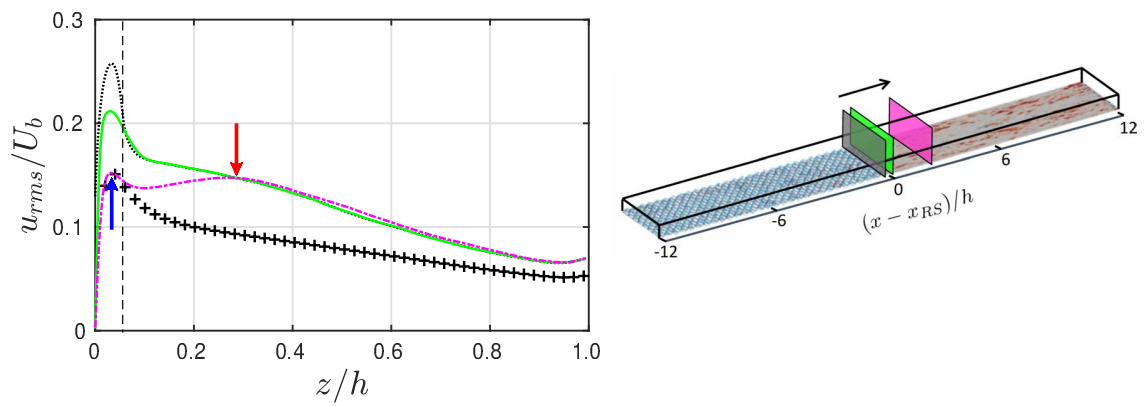

Figure 10: Profiles of $u_{r m s}$, normalised by $U_{b}$, at $\left(x-x_{R S}\right)=-0.05 h(\cdots \cdots), 0.05 h$ $(-)$, and $2 h(-\cdot-)$, indicated in the domain on the right. The vertical dashed line locates the roughness crest $(z=k)$. The upward and downward arrows indicate the inner and outer peaks of $(-\cdot-)$.

$\begin{array}{cccccc}\text { Study } & R e_{\tau_{r}}, R_{\tau_{s}} & k / h & k_{s}^{+} & \text {rough/smooth schematic figure } \\ \text { Case 24h } & 715,443 & 0.056 & 165 & \text { egg carton } \\ \text { Ismail et al. }(2018) & 2220,1277 & 0.083 & 1540 & \text { square bars } \square \square\end{array}$

Table 2: Summary of flow configuration for case $24 \mathrm{~h}$ and rough-to-smooth DNS of Ismail et al. (2018). The rough patch Reynolds number $R e_{\tau_{r}}=u_{\tau_{r}} h / \nu$ is computed at $\left(x-x_{R S}\right)=-h$, and the smooth patch Reynolds number $R e_{\tau_{s}}=u_{\tau_{s}} h / \nu$ is computed at $\left(x-x_{R S}\right)=7.5 h$. The arrow indicates the flow direction.

$U_{S}^{+}$by less than $1 \%, 2 \%$ and $5 \%$. It is seen that the recovered $z^{+}$between case $24 \mathrm{~h}$ and Ismail et al. (2018) does not change beyond a fetch of $\left(x-x_{R S}\right)=4.1 h$, despite the differences in $R e_{\tau}$ and roughness geometry. This finding is different than the wallmodelled LES of Saito \& Pullin (2014) over rough-to-smooth step change, and vice-versa. They showed that varying $R e_{\tau}$ by two orders of magnitude delays the recovery distance of $U^{+}$by two to three times. This difference might be due to the wider range of $R e_{\tau}$ in Saito \& Pullin (2014) or due to the wall-modelled LES, which inherently assumes some degree of equilibrium.

Table 3 shows that the equilibrium assumptions must be applied cautiously over the rough-to-smooth step change. For instance, to predict $u_{\tau}$ by at least $5 \%$ error from the equilibrium profile, the flow must be resolved down to $z^{+} \simeq 9$ at a fetch of $2.5 h$, and $z^{+} \simeq 69$ at a fetch of $7.5 h$. Beyond a fetch of $11 h$, fitting at any $z^{+}$yields $u_{\tau}$ with less than $5 \%$ error. Note that, these findings are based on the processed datasets and may change for other datasets.

\subsubsection{Smooth-to-rough step change}

Figure 13 shows the profiles of $U$ and $u_{r m s}$ up to a fetch of $2 h$ over the rough patch, zone R1. To measure the flow recovery, the profiles are compared against the DNS of homogeneous "egg-carton" rough open-channel flow, with $k / h=0.056$ at the expected fully recovered flow condition over the rough patch $\left(R e_{\tau}=704, L_{x} / h \times L_{y} / h \simeq 5.97 \times\right.$ $\left.3.18, \Delta_{x}^{+} \times \Delta_{y}^{+} \simeq 10.9 \times 5.8\right)$.

The $U^{+}$profiles (figure 13c), similar to the smooth patch, yield two logarithmic slopes with the inner one having a higher slope than the outer one. In figure $13(b, d, f)$ the 

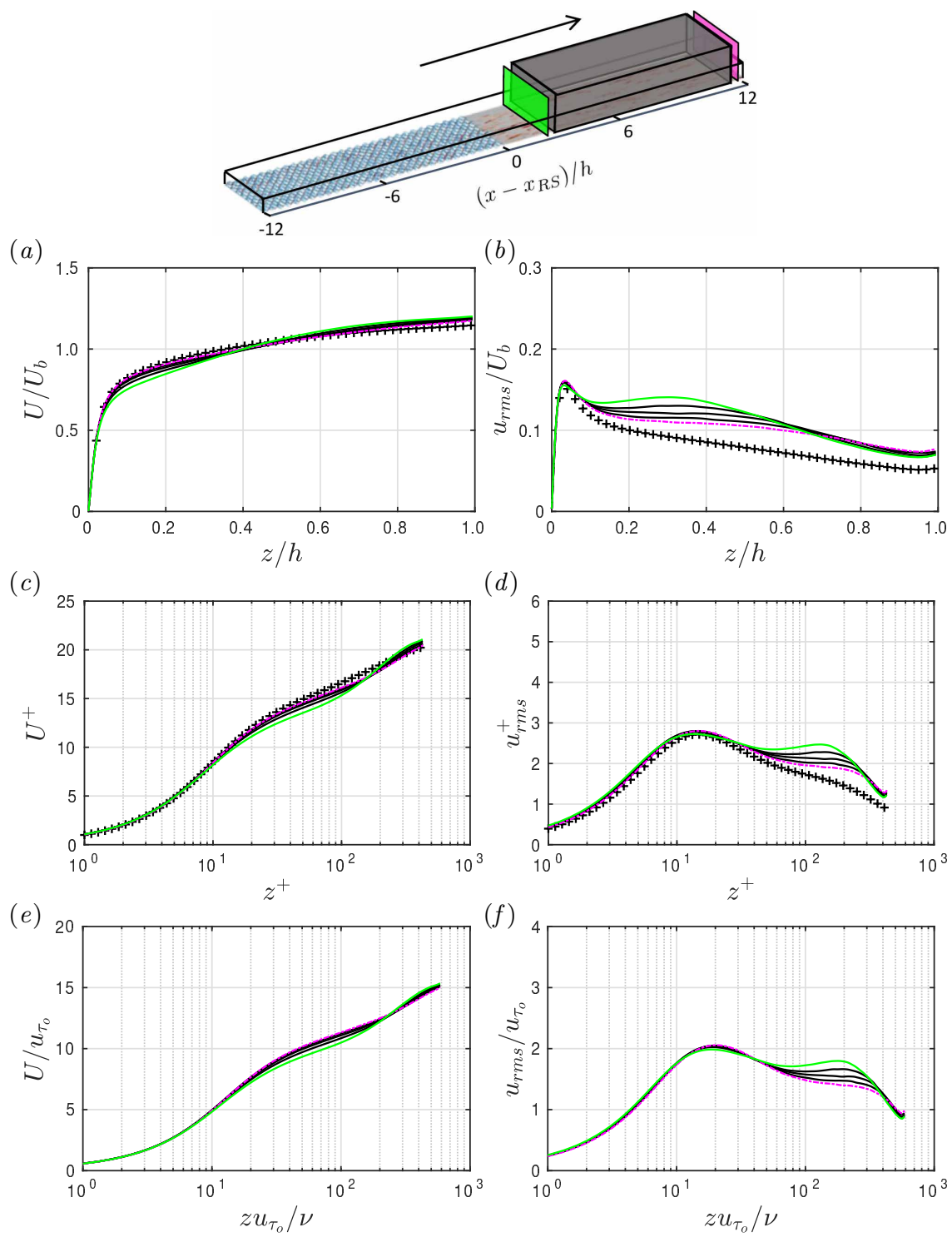

Figure 11: Profiles of $(a, c, e) U$, and $(b, d, f) u_{r m s}$ for $3 h \leq\left(x-x_{R S}\right) \leq 11 h$, zone S2. Normalisation is consistent with figure 8 . The black curves are equally spaced in the range $5 h \leq\left(x-x_{R S}\right) \leq 9 h$ (the shadowed box). $\left(x-x_{R S}\right)=3 h(-)$ and $11 h(-\cdot-)$. DNS of fully developed smooth open-channel flow at $R e_{\tau}=437(+)$.

$u_{r m s}$ profiles yield an inner peak below the roughness crest $(z / h \simeq 0.04, z / k \simeq 0.7)$. Chan et al. (2018) used a triple decomposition over the "egg-carton" roughness. They observed that the inner peak is due to the turbulent wakes behind the roughness elements. In the triple decomposition the fluctuations are decomposed into the coherent or timeaveraged spatially varying part $\widetilde{u}_{i}=\left\langle u_{i}\right\rangle_{t}-U_{i}$ (where $\langle.\rangle_{t}$ is averaged over time), and the background turbulence or time-varying part $u_{i}^{\prime}=u_{i}-\left\langle u_{i}\right\rangle_{t}$. As was shown in figure 10 (the green solid curve), the remnant of this inner peak persists in the $u_{r m s}$ at the beginning of the smooth patch. The $u_{r m s}$ inner peak over the rough patch does not change significantly 


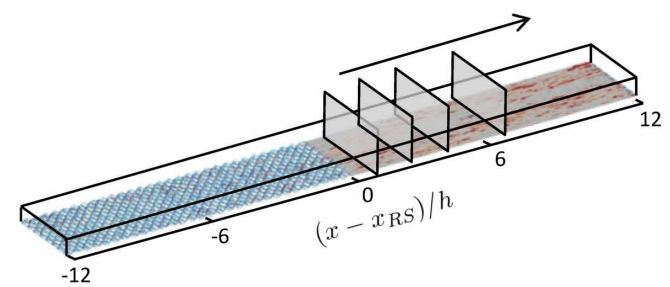

(a) $\left(x-x_{R S}\right)=0.8 h$

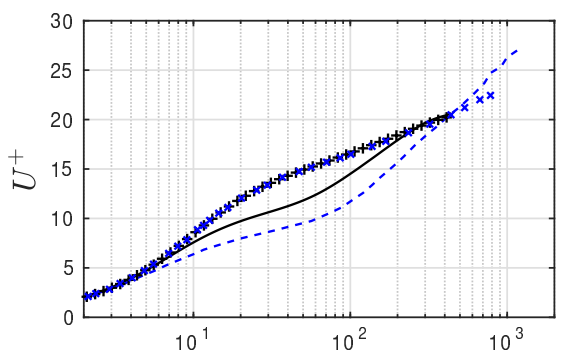

(c) $\left(x-x_{R S}\right)=4.1 \mathrm{~h}$

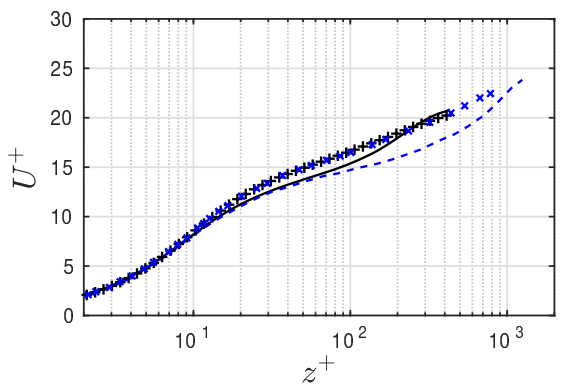

(b) $\left(x-x_{R S}\right)=2.5 h$

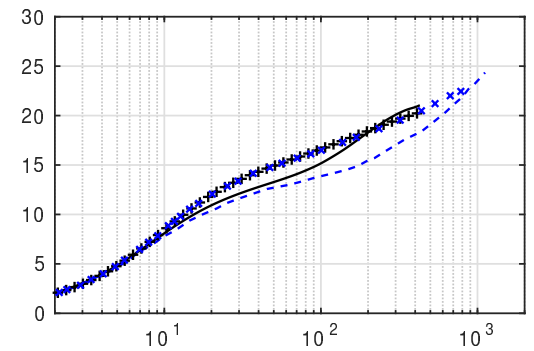

(d) $\left(x-x_{R S}\right)=7.5 h$

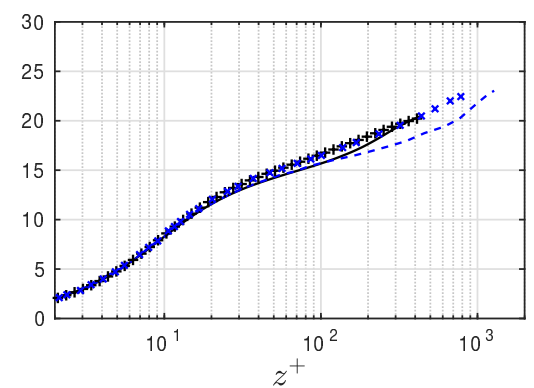

Figure 12: Rough-to-smooth comparison of $U^{+}$profiles between case $24 \mathrm{~h}(-)$ and DNS of Ismail et al. (2018) (-- - ). Comparison is made at the several $\left(x-x_{R S}\right)$ locations (shown in the domain): (a) $0.8 h ;(b) 2.5 h ;(c) 4.1 h ;(d) 7.5 h$. Fully recovered flow for case $24 \mathrm{~h}$ at $R e_{\tau}=437(+)$ and Ismail et al. $(2018)$ at $R e_{\tau}=1115(\times)$.

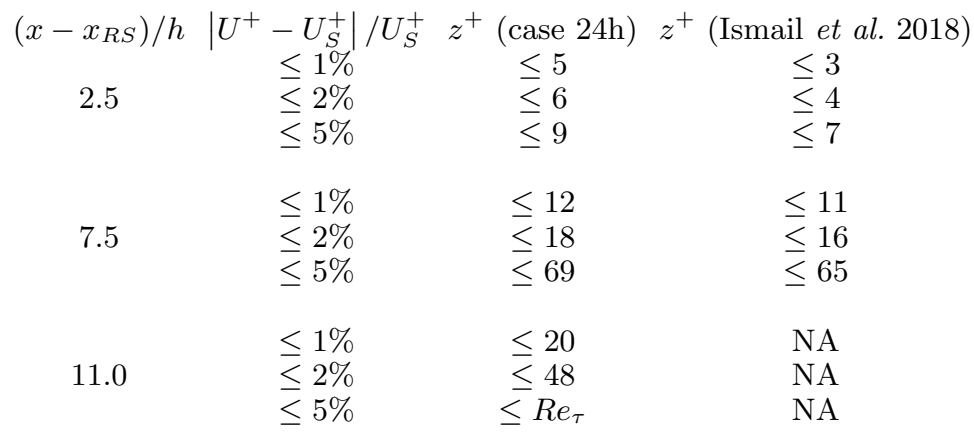

Table 3: Recovery in $U^{+}$of case $24 \mathrm{~h}$ and DNS of Ismail et al. (2018) after the rough-tosmooth step change. Recovery is measured based on $1 \%, 2 \%$ or $5 \%$ difference with the $U_{S}^{+}$profile of fully developed smooth channel. The fully developed case is at $R e_{\tau}=437$ for case $24 \mathrm{~h}$ and $R e_{\tau}=1115$ for Ismail et al. (2018). 

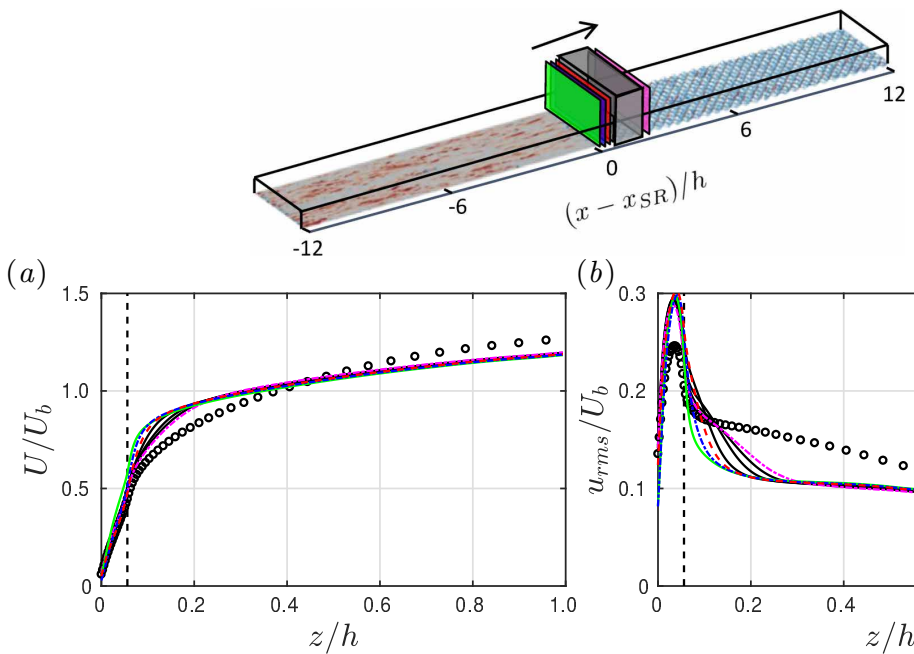

(b) (c)

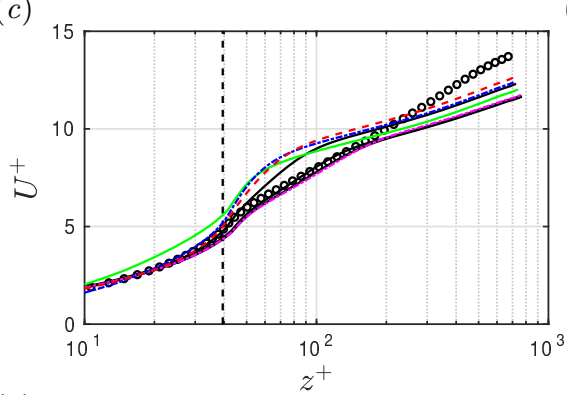

(e)

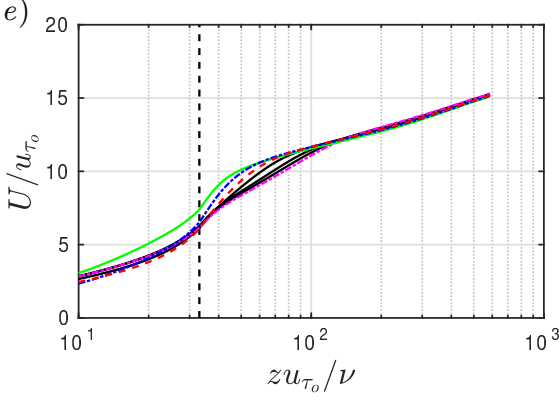

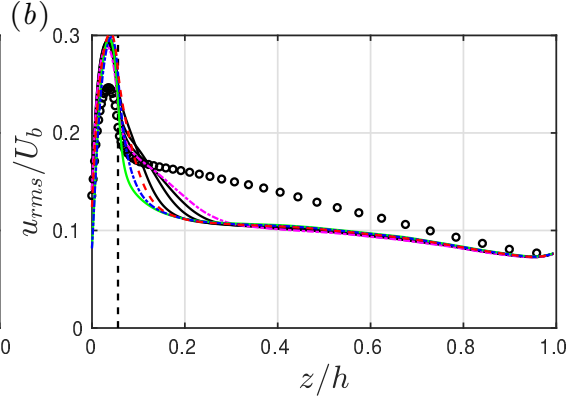

(d)

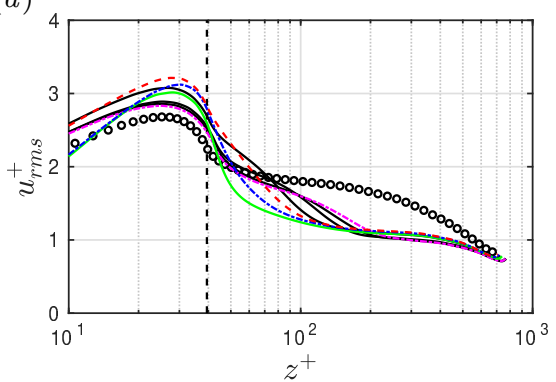

(f)

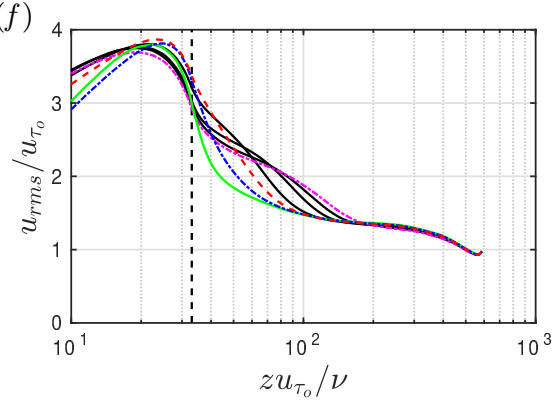

Figure 13: Profiles of $(a, c, e) U$, and $(b, d, f) u_{r m s}$ up to a fetch of $2 h$ over the rough patch, zone R1. Normalisation is consistent with figure 8 . The vertical dashed line locates the roughness crest. The black curves are equally spaced in the range $0.8 h \leq\left(x-x_{S R}\right) \leq 1.8 h$, $\left(x-x_{S R}\right)=0.2 h(-), 0.4 h(-\cdot-), 0.6 h(---)$ and $2 h(-\cdot-)$. Fully developed openchannel over homogeneous "egg-carton" roughness (०), with $k / h=0.056$ at $R e_{\tau}=704$.

up to a fetch of $2 h$ (figure $13 b, f$ ). This is not seen in the $u_{r m s}^{+}$profiles (figure $13 c$ ) because of their scaling by the variable local $u_{\tau}$. Beyond a fetch of $2 h$ (figure $14 b, f$ ) the $u_{r m s}$ inner peak gradually decreases. Above the roughness crest $(z / k>1)$, on the other hand, $u_{r m s}$ gradually increases along the rough patch (figure 13b,14b). Figure $7(e)$ showed the decrease of $u_{r m s}$ inner peak and its increase above the crest.

Profiles of $U$ and $u_{r m s}$ over the remainder of the rough patch, zone R2, are shown in figure 14. Compared to the smooth patch (figure 11) it appears that the profiles are recovered to a higher $z^{+}$after a fetch of $11 h$. The recovery over the rough patch is 

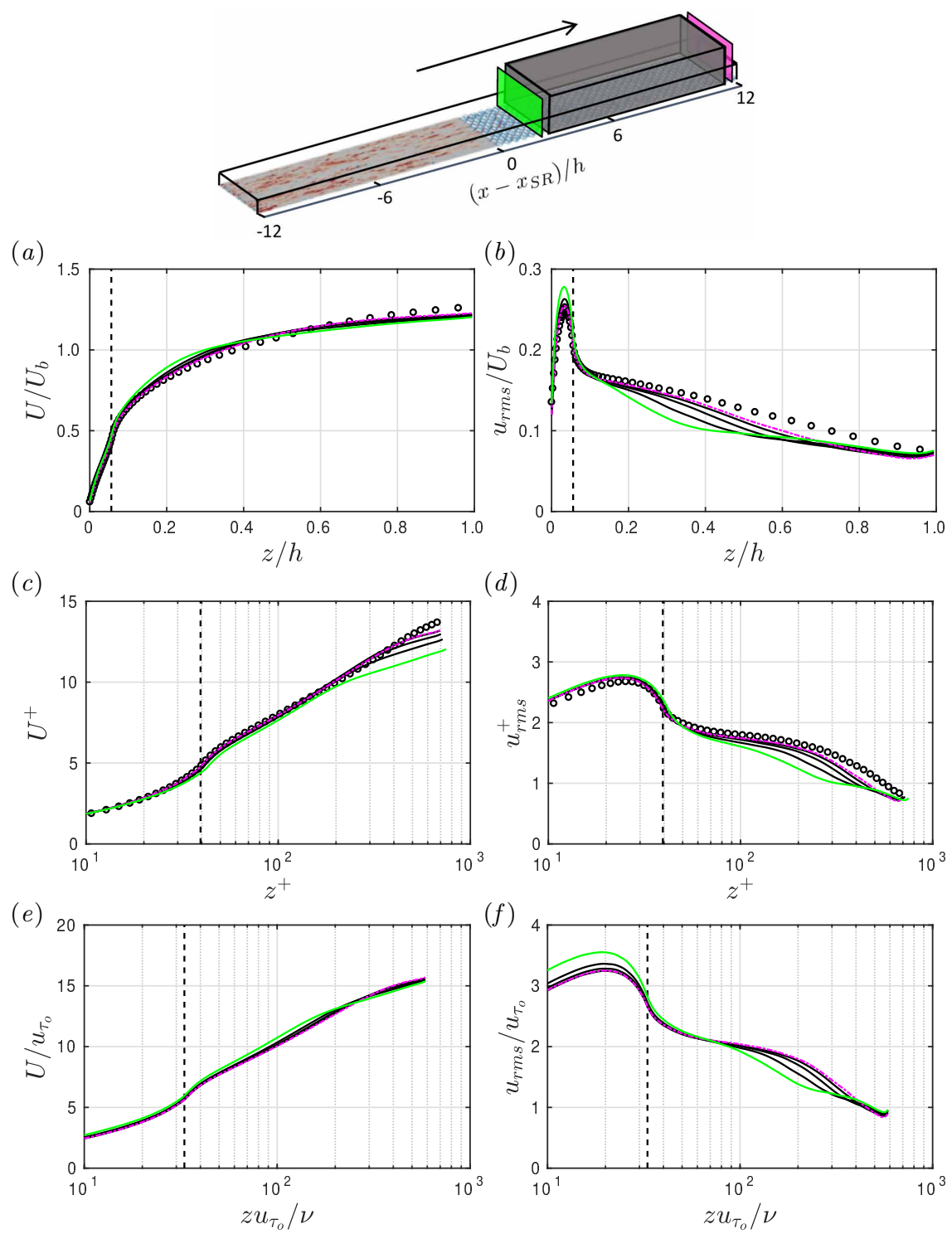

Figure 14: Profiles of $(a, c, e) U$, and $(b, d, f) u_{r m s}$ for $3 h \leq\left(x-x_{S R}\right) \leq 11 h$, zone R2. Normalisation is consistent with figure 8 . The black curves are equally spaced in the range $5 h \leq\left(x-x_{S R}\right) \leq 10 h,\left(x-x_{S R}\right)=3 h(-)$ and $11 h(-\cdot-)$. Fully developed openchannel over homogeneous "egg-carton" roughness (०), with $k / h=0.056$ at $R e_{\tau}=704$.

quantified in table 4, which confirms that recovery occurs faster compared to the recovery over the smooth patch (compare table 4 with table 3 ). Based on the $2 \%$ threshold, recovery in $U^{+}$over the rough patch (versus smooth patch) reaches up to $z^{+} \simeq 21$ (versus $z^{+} \simeq 6$ ) after a fetch of $2.5 h, z^{+} \simeq 475$ (versus $z^{+} \simeq 18$ ) after a fetch of $7.5 h$, and $z^{+} \simeq 528$ (versus $z^{+} \simeq 48$ ) after a fetch of $11 h$.

The study in this section yields the higher reliability of equilibrium assumptions over the rough patch than smooth patch. If an error up to $5 \%$ were considered acceptable and if the beginning of the log layer is classically noted as 30 wall units above the wall, 


$$
\begin{array}{cccc}
\left(x-x_{S R}\right) / h & \left|U^{+}-U_{R}^{+}\right| / U_{R}^{+} & z^{+} & z / k_{s} \\
& \leq 1 \% & \leq 19 & \leq 0.11 \\
2.5 & \leq 2 \% & \leq 21 & \leq 0.12 \\
& \leq 5 \% & \leq 28 & \leq 0.16 \\
& & & \\
& \leq 1 \% & & \leq 125 \leq 0.78 \\
7.5 & \leq 2 \% & \leq 163 \leq 1.01 \\
& \leq 5 \% & \leq R e_{\tau} \leq 4.35 \\
& \leq 1 \% & \leq 169 \leq 1.05 \\
11.0 & \leq 2 \% & \leq 528 \leq 3.28 \\
& \leq 5 \% & \leq R e_{\tau} \leq 4.35
\end{array}
$$

Table 4: Recovery in $U^{+}$of case $24 \mathrm{~h}$ after the smooth-to-rough step change based on the $1 \%, 2 \%$ or $5 \%$ difference with the $U_{R}^{+}$profile of fully developed homogeneous rough wall open-channel flow. The fully developed case is at $R e_{\tau}=704$ with the same roughness properties and channel height as the smooth-to-rough case. $k_{s} \approx 30 z_{0} \approx 4.1 k$.

over the rough patch the log-law assumption becomes valid (i.e. recovery reaches the beginning of the log layer) after a fetch of $2.5 \mathrm{~h}$. However, over the smooth patch the same assumption is valid only after a fetch of $5 h$. This conclusion is consistent with the results of Bou-Zeid et al. (2005) who compared wall-modelled LES of rougher-tosmoother transition (and vice-versa) with the field measurements of Bradley (1968). They observed large discrepancy with the field measurements at the initial $5 h$ distance after each step change. The discrepancy was decreased by further refining the grid and resolving the flow to a lower $z^{+}$.

\subsection{Internal boundary-layer}

This section studies the IBL and attempts to find a proper definition for its thickness $\delta_{i}$. The literature has not converged on a unified $\delta_{i}$ definition, and this consequently hinders a systematic comparison of the IBL growth rates. To demonstrate this divergence of views, some of the common definitions and the previous studies that have adopted these definitions are outlined in table 5 . The corresponding $R e_{\tau}$ in each study, as well as the obtained power-law exponents $\alpha$ for the IBL growth-rate $\left(\delta_{i} \propto x^{\alpha}\right)$ are added to the table.

The obtained power-law exponents in table 5 are compiled in figure 15. To ease the interpretation, the studies that have adopted the same definition are shown with the same symbol (and the same colour). Additionally, the results of some of the definitions that were applied to case $24 \mathrm{~h}$ are added to figure 15 , and are highlighted with circled symbols. At a fixed Reynolds number over either the smooth or rough patch, the obtained values of $\alpha$ from different definitions are substantially different than each other. This is also supported by the $50 \%$ variance seen in the resulting values of $\alpha$, obtained from case $24 \mathrm{~h}$. Thus, it appears that part of the scatter seen in figure 15 stems from the different definitions. Note that the studies highlighted with asterisks in table 5 considered transitions from a rougher to a smoother surface. Disregarding these studies from figure 15 (which correspond to some symbols for $R e_{\tau}>6 \times 10^{4}$ ), does not reduce the scatter caused by the IBL definition. In this section, the $\delta_{i}$ definitions arranged in table 5 are discussed further, through their application to case $24 \mathrm{~h}$. Eventually, a reliable definition is proposed according to the physical justifications. 


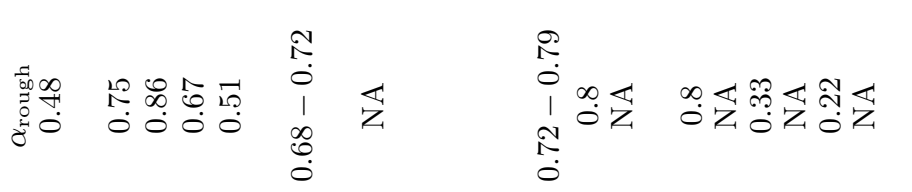

它宝言

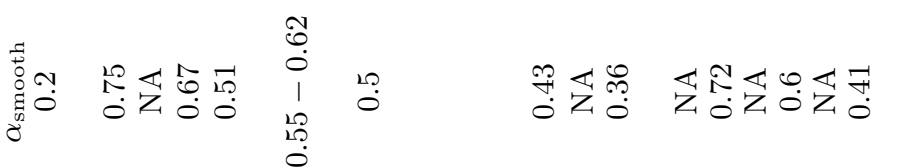

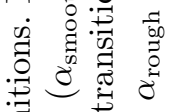

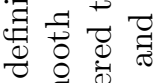

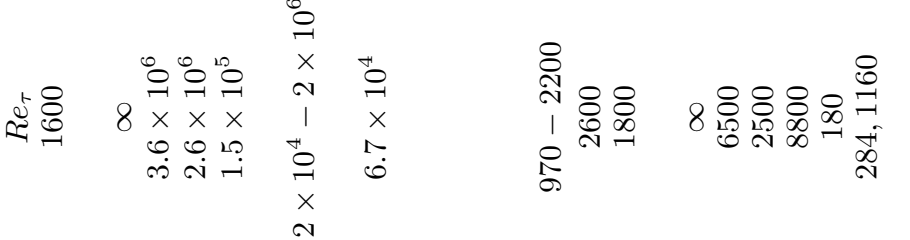

0 :

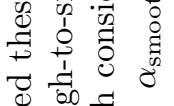

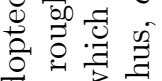

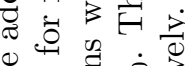

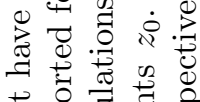

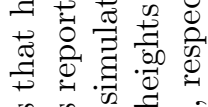

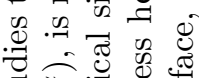

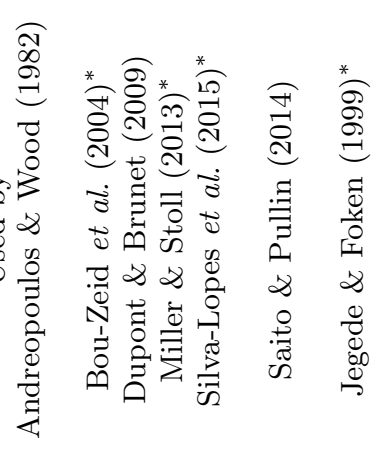

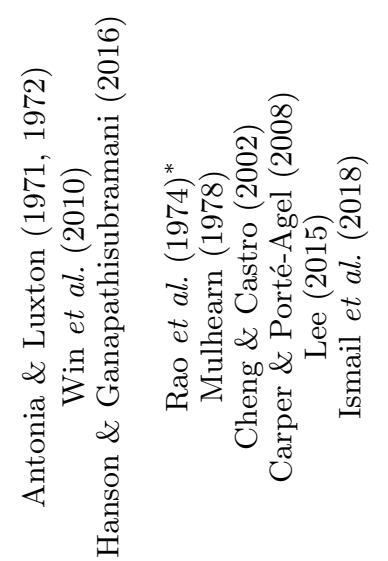

कै

品

.

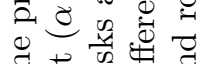

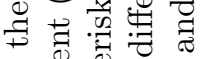

荧

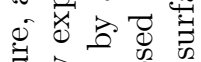

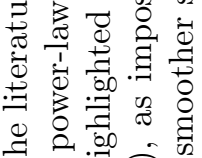

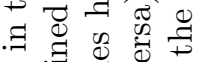

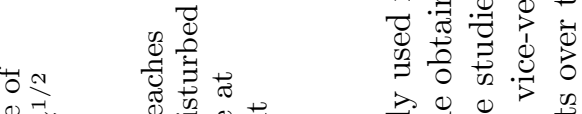

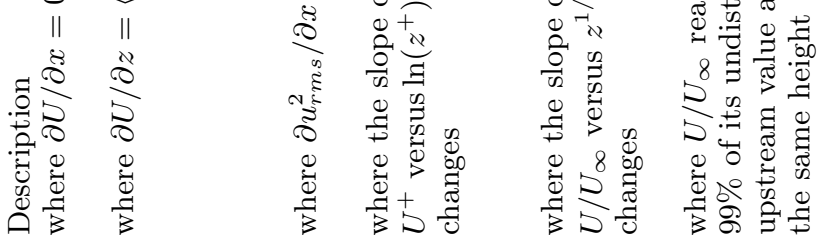

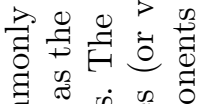

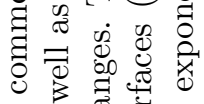

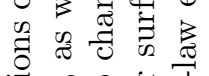

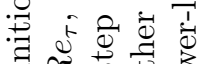

毒范范

io : :

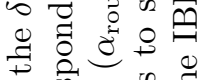

पै क्षै

항

ब

$\exists$

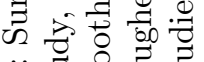

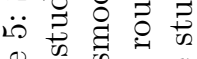

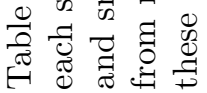


(a)

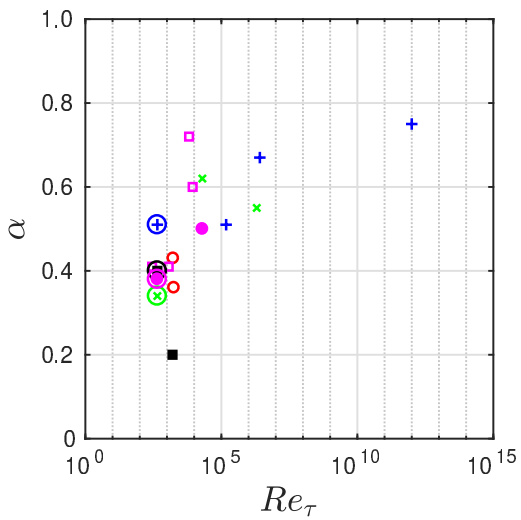

(b)

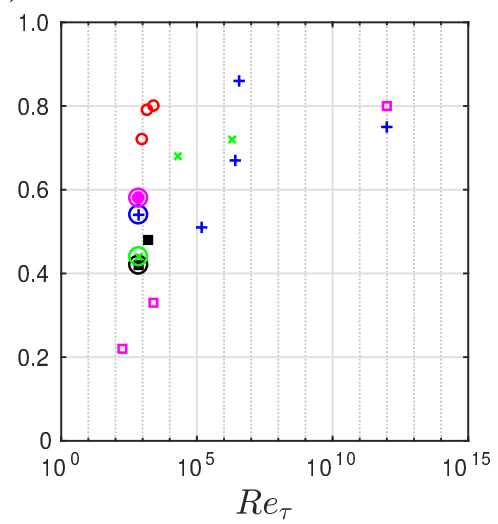

Figure 15: Values of the power-law exponent $\alpha$ for $\delta_{i}$ from the previous studies in table 5 . Studies that have adopted the same $\delta_{i}$ definition are indicated with the same symbol. (a) rough-to-smooth and (b) smooth-to-rough step change. AW (n); AL (o); BMP (+); $\mathrm{SP}(\mathrm{x}) ; \mathrm{E}(\bullet) ; \mathrm{PA}(\square)$. The circled symbols at $R e_{\tau}=443(a)$ and $R e_{\tau}=715(b)$ are obtained from application of different $\delta_{i}$ definitions on case $24 \mathrm{~h}$.

Figure 16 shows the application of the $\delta_{i}$ definitions (table 5 ) to case $24 \mathrm{~h}$. Each field in Figure 16 shows the characteristic parameter (e.g. $\partial U / \partial x, \partial u_{r m s} / \partial x$ ) to quantify $\delta_{i}$ base on each definition. All the definitions are invariant to the normalising velocity or length scale. The markers on each figure locate $\delta_{i}$ based on the corresponding definition. However, for definition AL (figure $16 e$ ) it is not trivial to identify $\delta_{i}$ from the current data. To recognise the different definitions, the subscript of $\delta_{i}$ shows the definition used from table 5 (e.g. $\delta_{B M P}$ is obtained from BMP, Bou-Zeid et al. 2004).

Figure 16(a) corresponds to $\delta_{A W}$ based on $\partial U / \partial x$. As was discussed in $\S 3.2$, $\partial U / \partial x$ is a measure of flow acceleration/deceleration. Originally this method was applied on boundary layer data, and a threshold was necessary for $\partial U / \partial x \simeq 0$. This is due to the unbounded nature of the boundary layer, in which the flow acceleration/deceleration near the wall is gradually decreased to zero away from the wall (Hanson \& Ganapathisubramani 2016). For the channel flow since $\partial U / \partial x$ changes its sign at some distance away from the wall ( $\S 3.2)$, detecting $\partial U / \partial x=0$ is straightforward. Figure $16(b, c)$ corresponds to $\delta_{B M P}$ and $\delta_{S P}$, respectively. $\delta_{B M P}$ is defined as the height where the local $\partial U / \partial z$ is equal to its $x$-averaged value, $\langle.\rangle_{x}$, and $\delta_{S P}$ is based on $\partial u_{r m s}^{2} / \partial x=0$.

Figure $16(d)$ shows the characterising parameter to identify $\delta_{E}$, defined based on the observation made in $\S 3.2$. The mean velocity profile after a surface change yields two logarithmic slopes. The inner slope is the result of the new surface, and the outer slope is the imprint of the previous surface. Elliott (1958) defines $\delta_{E}$ as the intersection point of inner and outer slopes. To detect the slopes in each $x$-location, the slope curve $\partial U^{+} / \partial \ln \left(z^{+}\right)$is plotted for the velocity profile at that location. This is demonstrated in figure 17 for a profile in the middle of the smooth patch (figure $17 a, c$ ), and for a profile in the middle of the rough patch (figure $17 b, d$ ). Note that the choice for scaling the profiles (here $u_{\tau}$ and $\nu$ ) does not affect the obtained $\delta_{E}$. The inner and outer logarithmic slopes appear as extrema in $\partial U^{+} / \partial \ln \left(z^{+}\right)$. Once the slopes (extrema) are found, two fitting lines with the same slopes are passed through the velocity profile at the located extrema. $\delta_{E}$ is identified by intersecting the two fitted lines. Application of this approach to the 
(a) $\left(h / U_{b}\right)(\partial U / \partial x)$

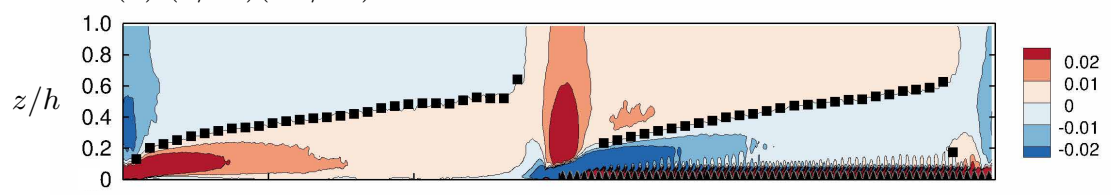

(b) $\left(h / U_{b}\right)\left(\partial U / \partial z-\langle\partial U / \partial z\rangle_{x}\right)$

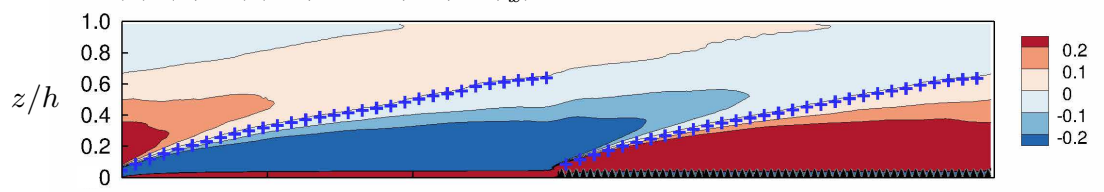

(c) $\left(h / U_{b}^{2}\right)\left(\partial u_{r m s}^{2} / \partial x\right)$

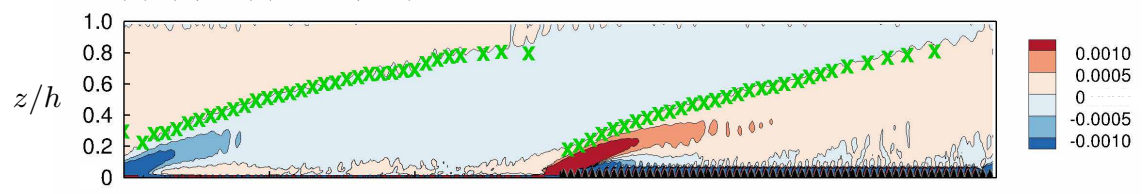

(d) $\partial U^{+} / \partial \ln \left(z^{+}\right)$

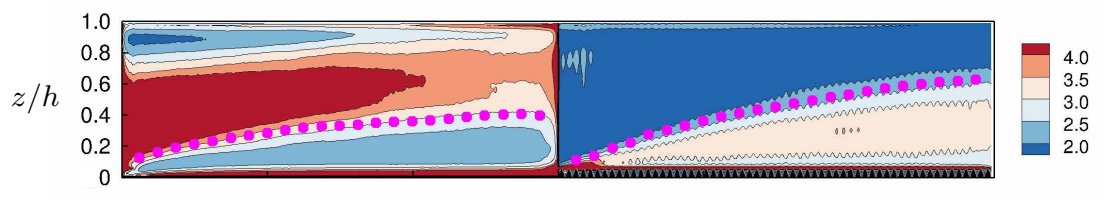

(e) $\partial\left(U / U_{b}\right) / \partial(z / h)^{1 / 2}$

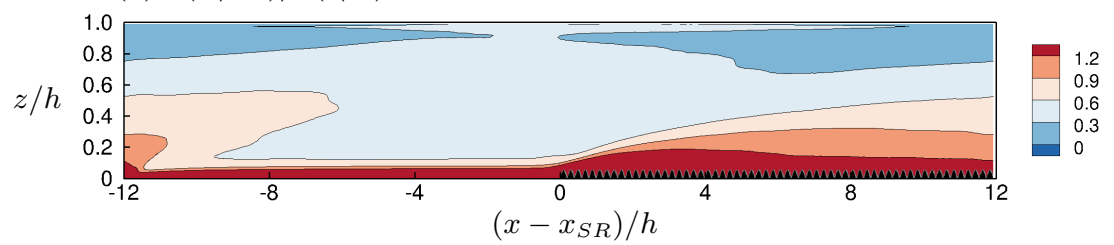

Figure 16: Application of the $\delta_{i}$ definitions in table 5 on case 24h. (a) AW, (b) BMP, $(c) \mathrm{SP},(d) \mathrm{E},(e) \mathrm{AL}$. In each figure the contour plot shows the characteristic parameter to identify $\delta_{i}$, and the symbols locate $\delta_{i}$ based on the corresponding definition. In $(e)$ detecting $\delta_{i}$ from AL was not straightforward (refer to text). $\delta_{A W}(\mathbf{\square}), \delta_{B M P}(+), \delta_{S P}$ $(\mathrm{x}), \delta_{E}(\bullet)$. The fields are overlaid by the spanwise projection of the roughness, in black colour.

whole field is shown in figure 16(d). The inner and outer slopes can be recognised as the two distinct (blue and red) regions. In addition to Elliott (1958), Panofsky \& Townsend (1964) proposed a variant of $\delta_{E}$ where $\delta_{i}$ was placed higher up, at the beginning of the upper logarithmic region. Here, the definition by Elliott (1958) is preferred as Panofsky \& Townsend's (1964) definition requires a threshold for the upper logarithmic region, while Elliott's (1958) definition, based on the intersection of the two logarithmic lines, is not threshold dependent.

The same slope-based approach was followed to calculate $\delta_{A L}$ (Antonia \& Luxton 1971). According to this definition, if the mean velocity is plotted against $z^{1 / 2}$, it yields two distinct straight-line slopes and $\delta_{A L}$ falls at their intersection. The profiles of $U / U_{b}$ versus $(z / h)^{1 / 2}$, over both the smooth and rough patches, are shown in figure 18 . The 


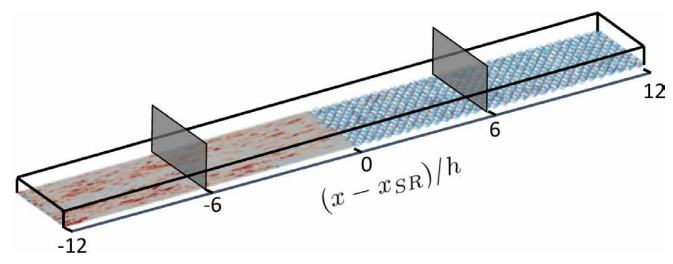

(a) $\left(x-x_{S R}\right)=-6.0 h$

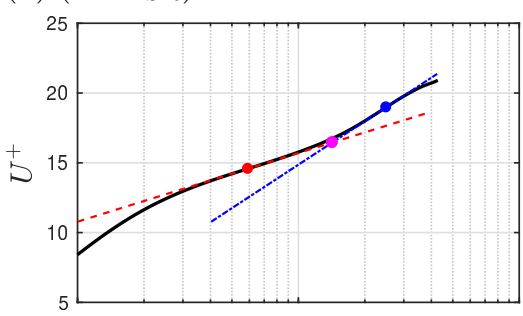

(c) $\left(x-x_{S R}\right)=-6.0 h$

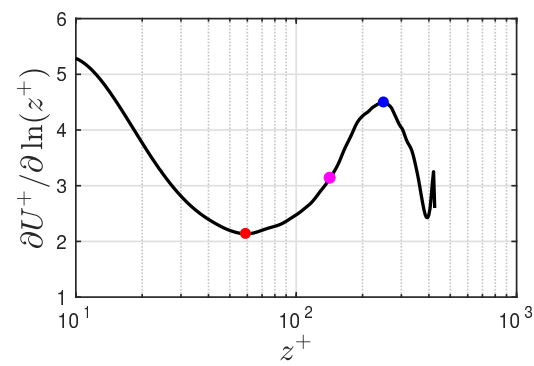

(b) $\left(x-x_{S R}\right)=6.0 h$

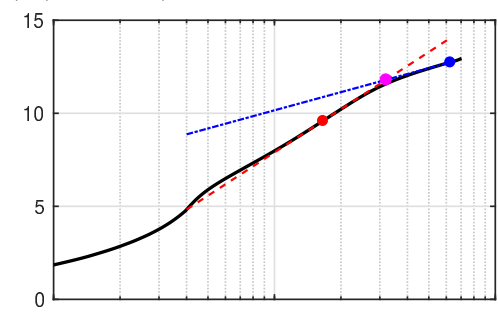

(d) $\left(x-x_{S R}\right)=6.0 h$

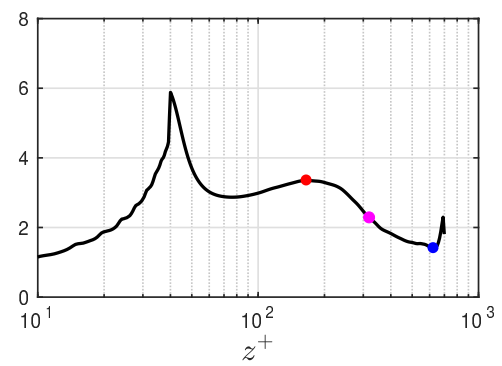

Figure 17: Identifying $\delta_{E}$ from logarithmic slope change. The quantities are normalised by the local $u_{\tau}$ and $\nu$. Profiles of $(a, b) U^{+}$at $\left(x-x_{S R}\right)=-6 h$ and $6 h$, indicated in the computational domain. Profiles of $(c, d) \partial U^{+} / \partial \ln \left(z^{+}\right)$corresponding to the profiles in $(a, b)$. The inner and outer slopes are the extrema of $\partial U^{+} / \partial \ln \left(z^{+}\right)(\bullet, \bullet) . \delta_{E}(\bullet)$ is located by intersecting the inner (---) and outer (---) logarithmic fitting lines.

profiles do not show two slopes. This is supported by the slope curves $\partial\left(U / U_{b}\right) / \partial(z / h)^{1 / 2}$ (figure $18 c, d$ ). Other than distinct peaks close to the wall, there are no clear extrema and no signs of the two distinct slopes that should be yielded by this technique. Figure 16(e) shows the characteristic parameter $\partial\left(U / U_{b}\right) / \partial(z / h)^{1 / 2}$ over the entire domain. Due to the gradual variation of this quantity, $\delta_{A L}$ is difficult to detect. The problem in applying this technique may lie with the lower Reynolds number of the current simulation. The experiments where this technique was applied extended up to and beyond $R e_{\tau} \simeq 2000$.

The method proposed by Pendergrass \& Arya (1984) is not applicable to channel flow as is quantified based on the velocity deviation from its undisturbed profile upstream of the surface change. In a channel flow there is a strong acceleration/deceleration during each surface change (figure $7 b$ ), that substantially modifies the $U$ profile across the entire channel. Hence the new profile is no longer comparable to the one upstream.

All $\delta_{i}$ values calculated from the different definitions are plotted in figure 19. Over the rough patch (figure $19 c, e$ ), with the exception of $\delta_{S P}(\mathrm{x})$, the three other definitions yield almost identical growth rates, especially for $\left(x-x_{S R}\right) \geq 4 h$. However, over the smooth patch discrepancy of up to $100 \%$ is seen among the definitions. Assessment of the obtained values of $\alpha$ (figure $19 d, e$ ) reveals their sensitivity to definition, which was earlier conjectured as one possible cause of scatter in the literature (figure 15). 
(a) $\left(x-x_{S R}\right)=-6.0 h$

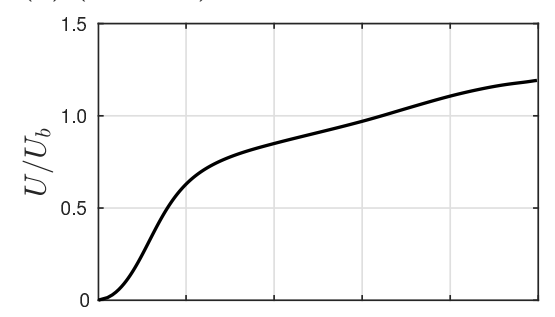

(c) $\left(x-x_{S R}\right)=-6.0 h$

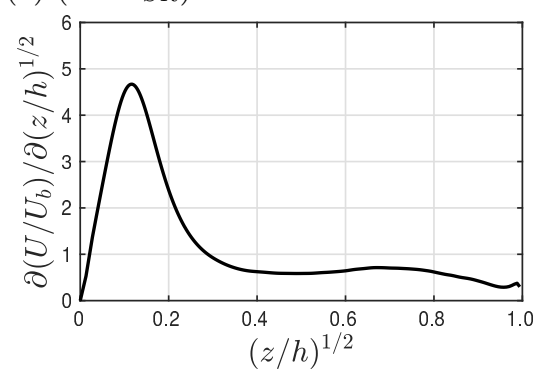

(b) $\left(x-x_{S R}\right)=6.0 \mathrm{~h}$

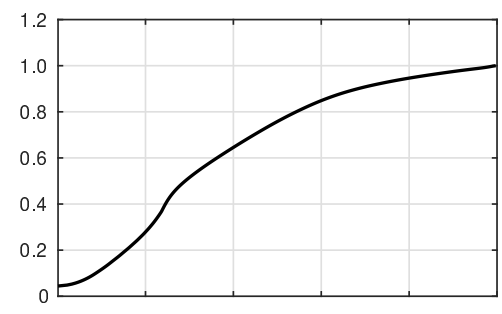

(d) $\left(x-x_{S R}\right)=6.0 h$

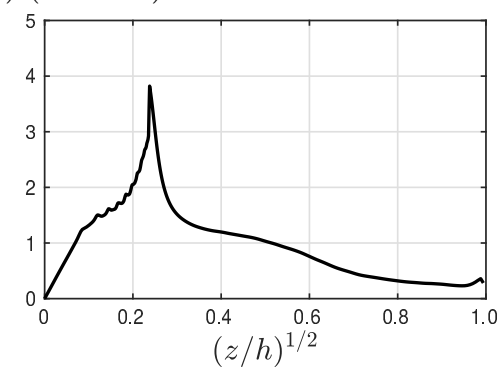

Figure 18: Profiles of $(a, b) U / U_{b}$ versus $(z / h)^{1 / 2}$ at the same locations indicated in figure 17. Profiles of $(c, d)$ the lope curves, $\partial\left(U / U_{b}\right) / \partial(z / h)^{1 / 2}$, corresponding to the profiles in $(a, b)$.

One notable behaviour in $\delta_{B M P}(+)$ is its almost identical growth rate over the smooth and rough patches, an observation that was earlier discussed by Bou-Zeid et al. (2004) and Silva-Lopes et al. (2015). Both Bou-Zeid et al. (2004) and Silva-Lopes et al. (2015) considered transitions from a rougher surface to a smoother surface (and vice-versa), with roughness height ratios of $z_{01} / z_{02}=10^{-1}$ and $z_{01} / z_{02} \approx 10^{-3}$, respectively. Despite the differences between the current DNS and these two studies, in each study $\delta_{B M P}$ yields the same power-law scaling over both smooth (or smoother) and rough (or rougher) surfaces, albeit with different power law scaling $\alpha$ between the studies.

One key finding from this section is the sensitivity of $\delta_{i}$ to its definition. This explains some of the discrepancies in the literature. The remainder of this section attempts to arrive at a physically motivated definition. The IBL definition must be consistent with the IBL concept, a layer that is influenced by the new surface and above which the flow does not feel the surface underneath. This concept also includes turbulence characteristics, i.e. turbulence characteristics within the IBL differ from those above. However, all the $\delta_{i}$ definitions in table 5 (except $\delta_{S P}$ ) are derived from the mean velocity. Therefore, a fair examination of consistency between the IBL concept and the IBL definitions would be through the turbulence characteristics.

Various definitions may be chosen to characterise turbulence. Here, the ratio of the turbulent time-scale over the mean time-scale $S^{*} \equiv|S| \mathcal{K} / \varepsilon$ (Pope 2000, § 7.1.7) is selected, where $\mathcal{K}$ and $\varepsilon$ are the turbulent kinetic-energy and its dissipation rate, respectively. $|S|=\sqrt{2 S_{i j} S_{i j}}$ is the mean strain-rate magnitude. In an equilibrium smooth channel flow at $R e_{\tau} \simeq 395, S^{*}$ is almost constant for $0.1 \leq z / h \leq 0.7$ (less than $\pm 10 \%$ variation). This range covers the heights above the buffer region up to the outer wake region. The constant $S^{*}$ is linked to the production-dissipation balance, and constancy of the normalised Reynolds shear-stress by $\mathcal{K}$ (Pope 2000). In figure $20 S^{*}$ is plotted, overlaid by the four IBL definitions. The region very close to the bottom 
(a) $U / U_{b}$

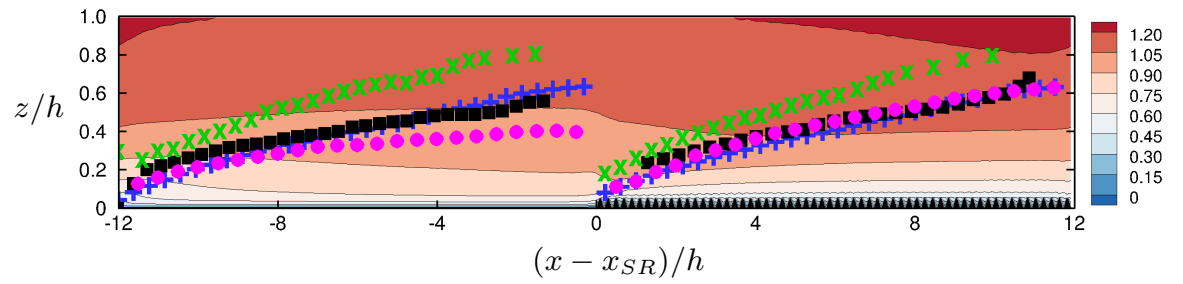

(b)

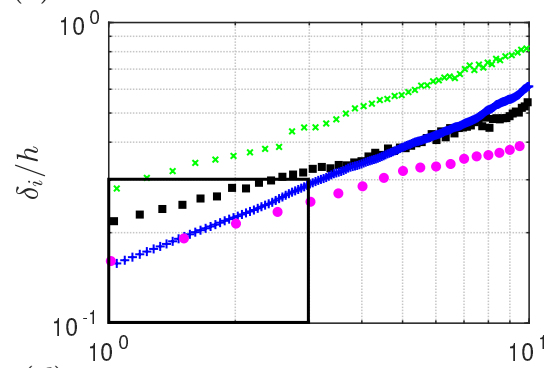

(d)

(c)
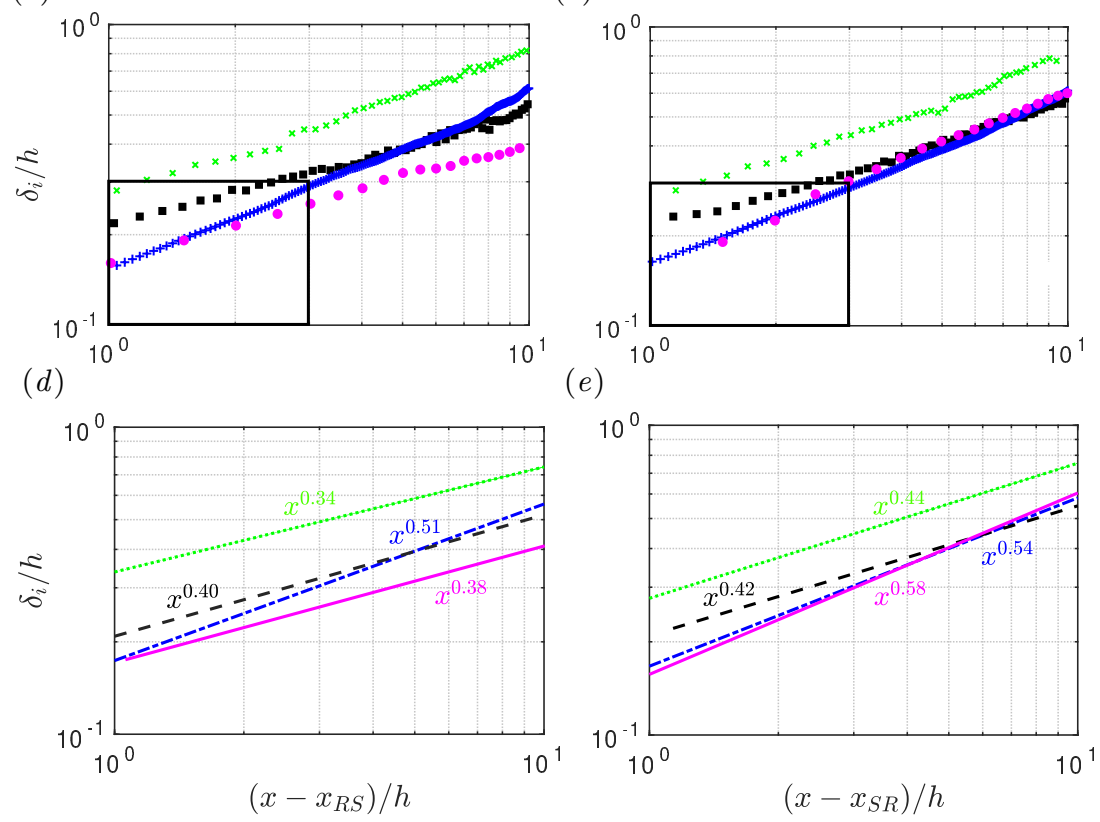

(e)

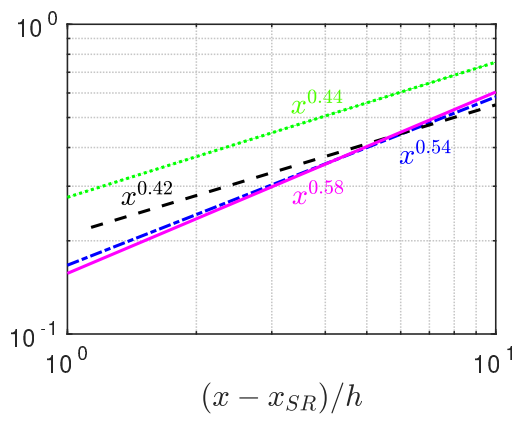

Figure 19: Comparison between the $\delta_{i}$ definitions throughout the domain in linear scale $(a)$. The resulting $\delta_{i}$ 's plotted in log scale over $(b)$ the smooth patch, and $(c)$ the rough patch. Note that, the origin has been shifted to the beginning of the smooth patch $\left(x_{R S}\right)$ in $(b, d)$. Power-law fitting the resulting $\delta_{i}$ 's over $(d)$ the smooth patch, and $(e)$ the rough patch. $\delta_{A W}(\bullet,---) ; \delta_{B M P}(+,-\cdot-) ; \delta_{S P}(\mathrm{x}, \cdots \cdots) ; \delta_{E}(\bullet,-)$. The framed regions in $(b, c)$ highlight the close behaviour of $\delta_{E}$ and $\delta_{B M P}$ within a fetch of $3 h$.

surface (the red region for $z / h \lesssim 0.1$ ) corresponds to the viscous and buffer regions, and is disregarded. Considering $0.1 \leq z / h \leq 0.6, S^{*}$ highlights two distinct regions, which differ in turbulence characteristics. The region closer to the wall is influenced by the new surface, while the region away from the wall preserves the characteristics associated with the previous surface. Among all the IBL definitions, $\delta_{E}$ appears to behave more consistently with the distinct regions created by $S^{*}$. Over the rough patch all the IBL definitions, except $\delta_{S P}$, behave consistent with the distinct regions. However, over the smooth patch only $\delta_{E}$ captures the sharp gradient in $S^{*}$ that marks the edge of the internal boundary layer. As a support to the latter argument, the gradient magnitude of $S^{*},\left|\nabla S^{*}\right|=\sqrt{\left(\partial S^{*} / \partial x\right)^{2}+\left(\partial S^{*} / \partial z\right)^{2}}$, is plotted in figure 21. The regions where this quantity is maximum corresponds to the regions where $S^{*}$ has the largest variation (i.e. turbulence characteristics are changing). As is seen in figure 21(a), the regions of maximum $\left|\nabla S^{*}\right|$ appear as layers that are emanated from the leading edges of the smooth 
(a) $\delta_{A W}$

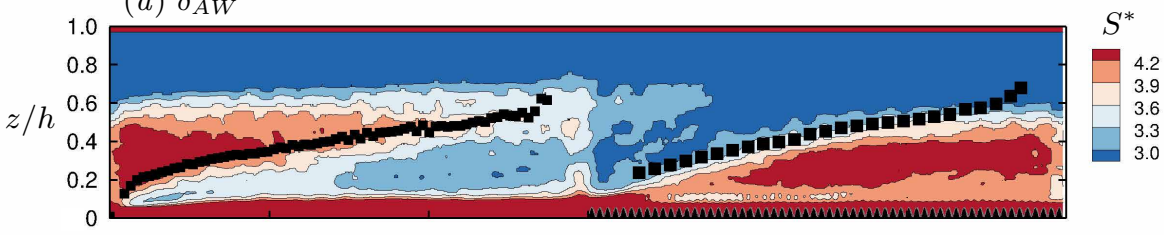

(b) $\delta_{B M P}$

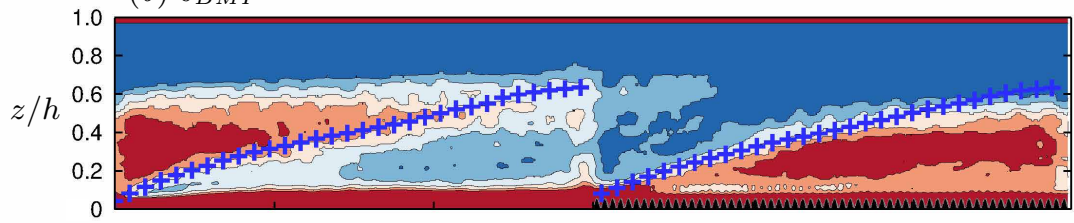

(c) $\delta_{S P}$

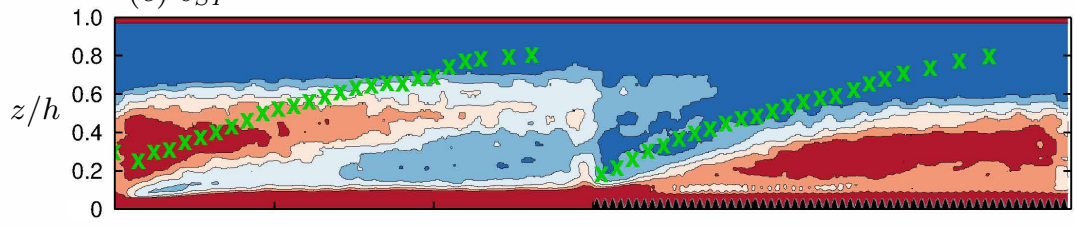

$(d) \delta_{E}$

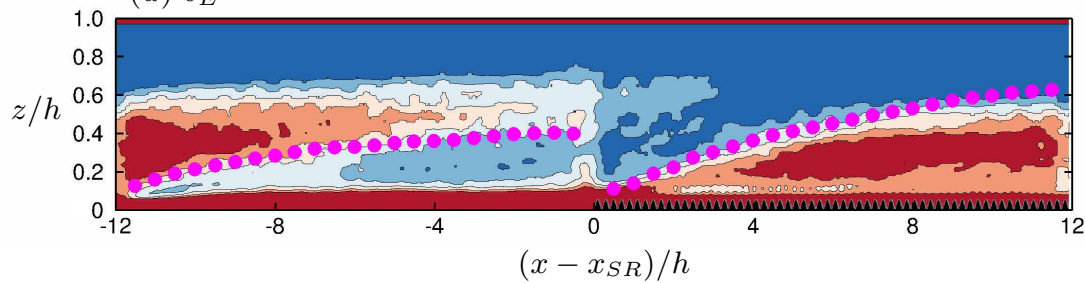

Figure 20: The normalised mean shear-rate $S^{*}=|S| \mathcal{K} / \varepsilon$, overlaid with the IBL definitions. $(a) \delta_{A W}(\bullet),(b) \delta_{B M P}(+),(c) \delta_{S P}(\mathrm{x})$, and $(d) \delta_{E}(\bullet)$. The fields are overlaid by the spanwise projection of the roughness, in black colour.
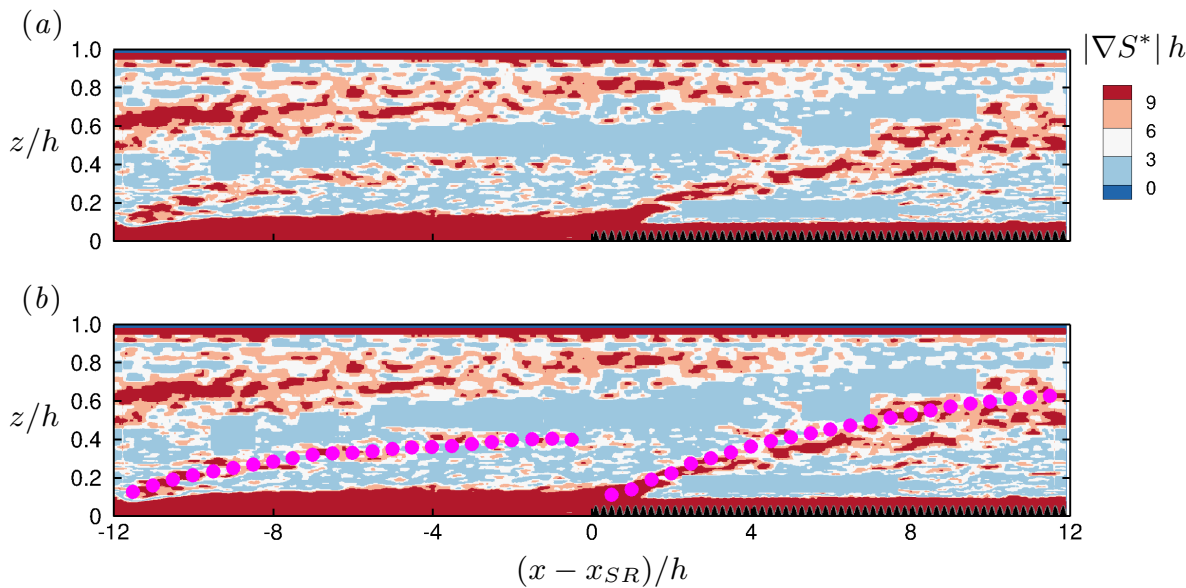

Figure 21: The gradient magnitude of $S^{*}\left(\left|\nabla S^{*}\right|\right)$, corresponding to figure 20 is shown in $(a)$ and $(b)$. In $(b)\left|\nabla S^{*}\right|$ is overlaid with $\delta_{E}(\bullet)$. The fields are overlaid by the spanwise projection of the roughness, in black colour. 


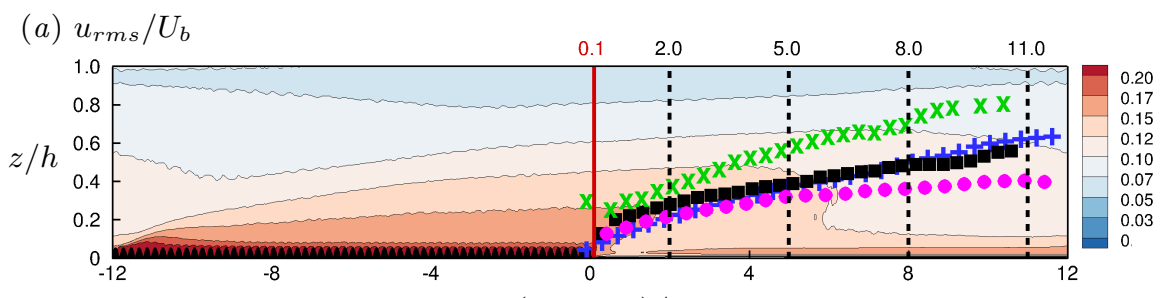

$$
\left(x-x_{R S}\right) / h
$$
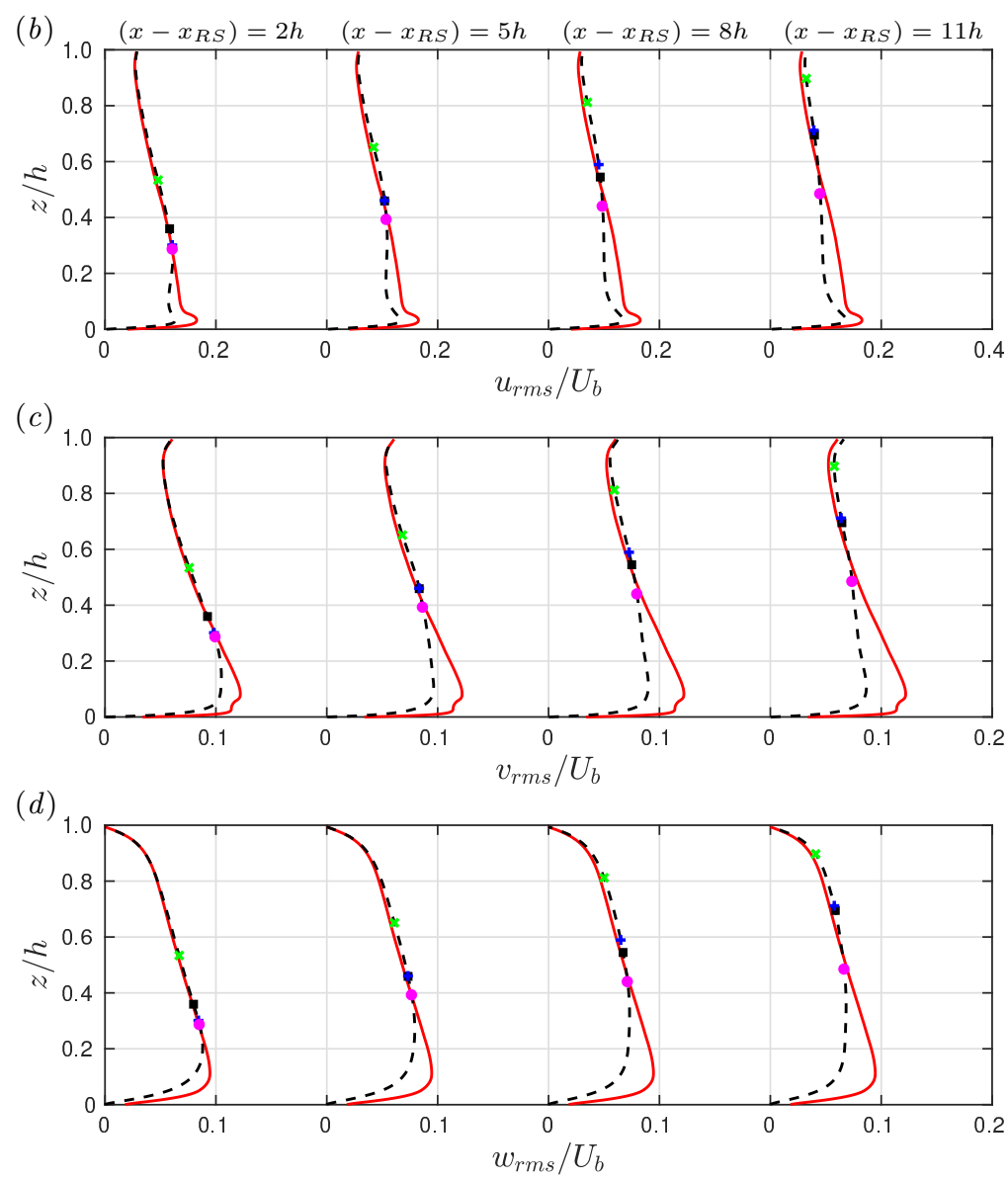

Figure 22: (a) $u_{r m s}$ field overlaid with the IBL definitions. Comparison of profiles of (b) $u_{r m s},(c) v_{r m s}$, and $(d) w_{r m s}$, after a fetch of $2 h, 5 h, 8 h, 11 h$ over the smooth patch $(---)$, with their upstream counterparts after a fetch of $0.1 h(-)$. The extracted locations are indicated in the $u_{r m s}$ field in $(a)$. The four overlaid IBL definitions are: $\delta_{A W}(\boldsymbol{\bullet}), \delta_{B M P}(+), \delta_{S P}(\mathrm{x})$, and $\delta_{E}(\bullet)$. The spanwise projection of the "egg carton" roughness is indicated with black colour.

and rough patches. In figure $21(b)$ it is seen that over each patch, $\delta_{E}$ is coincident with this newly formed layer of maximum $\left|\nabla S^{*}\right|$ that is emanated from the leading edge of each patch.

Consistency of the IBL definitions with the IBL concept is examined through the r.m.s. quantities over the smooth patch in figure 22. Profiles of $u_{r m s}$ (figure $22 b$ ), $v_{r m s}$ 
(figure $22 c$ ) and $w_{r m s}$ (figure $22 d$ ), at a fetch of $2 h, 5 h, 8 h$ and $11 h$ over the smooth patch (---) are compared with their upstream counterparts immediately downstream of the rough-to-smooth step change, at a fetch of $0.1 \mathrm{~h}(-)$. At each downstream location the height up to which the r.m.s. profile departs from its upstream counterpart indicates the maximum height to which the new surface influence has reached (i.e. IBL concept based on r.m.s. quantities). Figure 22 shows that among the four overlaid $\delta_{i}$ definitions, $\delta_{E}(\bullet)$ coincides better with the departure point for all three r.m.s. quantities; this is more evident in the most downstream profile at $\left(x-x_{R S}\right)=11 h$. The same study was conducted over the rough patch (not shown) and with the exception of $\delta_{S P}$, the three other definitions agreed well with the departure point. Therefore, from turbulence characteristics and r.m.s. quantities it is concluded that $\delta_{E}$ is more consistent with the IBL concept. Based on either turbulence characteristics $S^{*}$ (figures 20,21) or any of the r.m.s. quantities (figure 22), Elliott's (1958) definition $\left(\delta_{E}, \bullet\right)$ is consistent with the IBL concept. Therefore, any new IBL definition that is derived from $S^{*}$ or Reynolds stresses would be no different than $\delta_{E}$.

This section represents the first to analyse the IBL definitions so extensively, highlighting the large discrepancy in IBL growth rates from the various definitions for the same flow. We can thus propose the most physically consistent IBL definition.

\section{Conclusions}

In this study DNSs of open-channel flow over streamwise-alternating patches of smooth and fully rough walls were investigated. The computational domain was equally divided between the smooth patch and the rough patch. Owing to the streamwise periodicity, both rough-to-smooth and smooth-to-rough step changes were studied. With the detailed information provided by DNS, some aspects of this flow were investigated that were hard to explore through either experimental techniques or computational models. These aspects included: (1) the validity of the equilibrium assumptions, and (2) a thorough study on the internal boundary-layer (IBL) definitions.

Before studying the above-mentioned aspects, it was ensured that the parameters of interest are invariant to the finite domain size, and its periodicity. To this aim, three cases with domain lengths varying from 6 to 24 times the channel height $h$, as well as a nonperiodic rough-to-smooth case with fully developed inflow were simulated. The results showed that with a domain length of at least $12 h$ (assigning $6 h$ to each patch), the flow quantities within the IBL are not influenced by the domain length and periodicity. Above the IBL, due to the history effects, the flow remains sensitive. Nevertheless, the physics of interest occur within the IBL or at its edge, including: the wall shear-stress, the IBL thickness and the flow recovery.

Assessment of the mean velocity profiles revealed that the equilibrium assumptions are not entirely valid, in particular over the smooth patch. If an error up to $5 \%$ is noted acceptable and if the beginning of the log-layer is classically noted as 30 wall units above the wall, over the rough patch the log-law assumption becomes valid after a fetch of $2.5 h$, while over the smooth patch is valid after a fetch of $5 h$.

An extensive study was conducted on the IBL. Most commonly used definitions of the IBL thickness were tested on the current DNS. It was noticed that for the same dataset, depending on the definition, the resulting IBL thickness may differ by up to $100 \%$. To choose the proper definition, the authors started from the fundamental perception of the IBL, as a layer that separates the influenced region by the surface underneath from the uninfluenced one. Then, they applied this concept to the turbulence characteristics and r.m.s. quantities. Results showed that the definition by Elliott (1958) that is based 
on the logarithmic slope change of the velocity profile, is more consistent with this perception of the IBL.

\section{Acknowledgements}

This research was supported by resources provided by The Pawsey Supercomputing Centre with funding from the Australian Government and the Government of Western Australia and by the National Computing Infrastructure (NCI), which is supported by the Australian Government. This research was partially supported under the Australian Research Councils Discovery Projects funding scheme (project DP160103619). The authors acknowledge the helpful comments by Prof. E. Bou-zeid and the two other anonymous referees.

\section{Appendix A. Numerical scheme and the immersed boundary method}

In this Appendix details of the numerical scheme, the immersed boundary method (IBM), and verification of the numerical setup against a body-conforming grid solver are presented.

Equation (2.1) in $\S 2$ is integrated in time using the fractional-step algorithm (Perot 1993). The time-marching scheme is the third-order Runge-Kutta (Spalart et al. 1991), which divides each time-step into three sub-steps. During each sub-step, the fractionalstep algorithm consists of the following three steps to update the velocity from the current sub-step $\left(u_{i}^{n}\right)$ to the next sub-step $\left(u_{i}^{n+1}\right)$ :

1) Predicting the intermediate velocity $\left(u_{i}^{*}\right)$ :

$$
\frac{u_{i}^{*}-u_{i}^{n}}{\Delta_{t}}=\text { Explicit }+\nu\left(\frac{\partial^{2} u_{i}}{\partial x_{3}^{2}}\right)^{n, *}+F_{i}^{m}
$$

$$
\text { Explicit }=\xi_{m}\left[-\frac{1}{\rho}\left\langle\frac{d P}{d x}\right\rangle \phi_{u} \delta_{i 1}-\frac{1}{\rho}\left(\frac{\partial \widetilde{p}}{\partial x_{i}}\right)^{n}\right]+\left[\nu\left(\frac{\partial^{2}}{\partial x_{1}^{2}}+\frac{\partial^{2}}{\partial x_{2}^{2}}\right) u_{i}-\frac{\partial u_{i} u_{j}}{\partial x_{j}}\right]^{n, n-1}
$$

2) Solving the Poisson equation:

$$
\frac{1}{\rho} \frac{\partial^{2}[\delta \widetilde{p}]}{\partial x_{j}^{2}}=\frac{1}{\xi_{m} \Delta_{t}} \frac{\partial u_{i}^{*}}{\partial x_{i}}
$$

3) Updating the velocity $\left(u_{i}^{n+1}\right)$ and periodic pressure $\left(\widetilde{p}^{n+1}\right)$ for the next sub-step:

$$
u_{i}^{n+1}=u_{i}^{*}-\frac{\xi_{m} \Delta_{t}}{\rho} \frac{\partial[\delta \widetilde{p}]}{\partial x_{i}}, \quad \widetilde{p}^{n+1}=\widetilde{p}^{n}+\phi_{p}[\delta \widetilde{p}]
$$

The spatial discretisation is the fully conservative fourth-order, staggered, finitedifference scheme (Morinishi et al. 1998; Verstappen \& Veldman 2003). $\xi_{m} \Delta_{t}$ is one sub-step size, i.e. $\Delta_{t}=\xi_{1} \Delta_{t}+\xi_{2} \Delta_{t}+\xi_{3} \Delta_{t}$. The advection and the wall-parallel diffusion terms are advanced explicitly using the low-storage third-order Runge-Kutta, $(.)^{n, n-1}=\gamma_{m}(.)^{n}+\zeta_{m}(.)^{n-1}$, while the wall-normal diffusion term is advanced implicitly, $(.)^{n, *}=\alpha_{m}(.)^{n}+\beta_{m}(.)^{*}$, where $\alpha_{m}, \beta_{m}, \gamma_{m}$, and $\zeta_{m}$ depend on the sub-step (Spalart et al. 1991, its appendix).

To impose the no-slip condition on the bottom smooth and rough surfaces, the IBM force:

$$
F_{i}^{m}=-\left(1-\phi_{i}\right)\left[\text { Explicit }+\nu\left(\partial^{2} u_{i} / \partial x_{3}^{2}\right)^{n, *}+u_{i}^{n} / \Delta_{t}\right]
$$

is added such that $u_{i}^{*}=0$ when $\phi_{i}=0$, as written in (A 1). $\phi_{i}$ is the fraction of 


\section{Case $\quad \begin{array}{llllllllll}L_{x} / h & L_{y} / h & N_{x} & N_{y} & N_{z} & \Delta_{x_{s}}^{+}, \Delta_{x_{r}}^{+} & \Delta_{y_{s}}^{+}, \Delta_{y_{r}}^{+} & \lambda / \Delta_{x} & \lambda / \Delta_{y}\end{array}$ $\begin{array}{llllllllll}\text { 6h-verification } & 6.06 & 3.18 & 384 & 384 & 192 & 6.8,12.3 & 3.7,6.2 & 24.0 & 48.0\end{array}$}

Table 6: Summary of the verification case using the body-conforming grid solver (Cascade Technologies, Inc. Ham et al. 2006) for comparison with case $6 \mathrm{~h}$ in table 1 using the immersed-boundary method. The global Reynolds number $R e_{\tau_{o}}=590$, the same as case 6 h.

each computational cell occupied by the fluid, computed during pre-processing for the staggered velocity components and pressure $\left(\phi_{u}, \phi_{v} \phi_{w}\right.$ and $\left.\phi_{p}\right)$. Through substitution for $F_{i}^{m}$ in (A 1), step (1) can be recast into the following equation:

$$
\left(1-\nu \phi_{i} \beta_{m} \Delta_{t} \frac{\partial^{2}}{\partial x_{3}^{2}}\right) u_{i}^{*}=\phi_{i}\left(1+\nu \alpha_{m} \Delta_{t} \frac{\partial^{2}}{\partial x_{3}^{2}}\right) u_{i}^{n}+\Delta_{t} \phi_{i} \text { Explicit }
$$

The term in the brackets, on the left hand side of (A.5) is a heptadiagonal matrix (owing to the fourth-order discretisation), which is solved directly for $u_{i}^{*}$.

The wall shear-stress at each time step at each $(x, y)$ location can be obtained through integration of the streamwise IBM force term $F_{1}^{m}$ over the $z$-direction:

$$
\frac{\tau_{w}}{\rho}=-\sum_{m=1}^{3}\left[\int_{z_{\min }}^{z_{\max }} F_{1}^{m} \mathrm{~d} z\right]
$$

where the integrals are summed over the three sub-steps of Runge-Kutta (i.e. $m=1,2,3$ ), and $z_{\min }$ and $z_{\max }$ are the minimum and maximum $z$-coordinates of the computational domain (here $-4 / 3 k$ and $h$, respectively). The friction velocity $u_{\tau}(x)=\sqrt{\left\langle\tau_{w}\right\rangle / \rho}$, is obtained through averaging $\tau_{w}$ over time and spanwise direction. Over the rough patch $\langle$.$\rangle also indicates averaging over a finite streamwise window size, following the procedure$ described in $\S 2$.

This IBM is of the direct-forcing category with the Volume of Fluid (VOF) interpolation (Fadlun et al. 2000) suitable to implement solid geometries in Cartesian codes. In this method the computational domain includes both the solid and fluid regions (figure $2 a$ ), from $z_{\min }=(-4 / 3) k$ to $z_{\max }=h$, placing a $(1 / 3) k$ solid gap between the roughness trough and the bottom computational boundary, with the no-slip condition imposed on the bottom boundary. To drive only the fluid zone, $\langle d P / d x\rangle$ has been multiplied by $\phi_{u}$ in (A 1). The direct-forcing IBM with the VOF interpolation has been adopted in the previous rough-wall simulations (Scotti 2006; Yuan \& Piomelli 2014). However, the IBM adopted here has been slightly modified compared to the one adopted by Scotti (2006) and Yuan \& Piomelli (2014) by adding (A.3b) in step (3), which corrects the pressure by $\phi_{p}$. With this modification, $F_{i}^{m}$ is non-zero only in the computational cells that intersect the solid-fluid interface. However, in the uncorrected approach $F_{i}^{m}$ is also non-zero in the non-intersecting in-solid cells. The modified approach yields $u_{\tau}$ more directly in heterogeneous flows.

The code that adopts the numerical schemes described above, without the IBM, has been verified in the previous DNS studies (Chung et al. 2014, 2015). To verify the IBM, a grid-refinement study and a comparison against a body-conforming grid solver were carried out. The grid-refinement study was conducted for homogeneous "egg carton" roughness (implemented with the IBM), at $R e_{\tau}=590$, and grid convergence was reached when $\lambda / \Delta_{x}=25.6$ and $\lambda / \Delta_{y}=48.0$. Then case $6 \mathrm{~h}$ (table 1 ) was repeated using a body- 

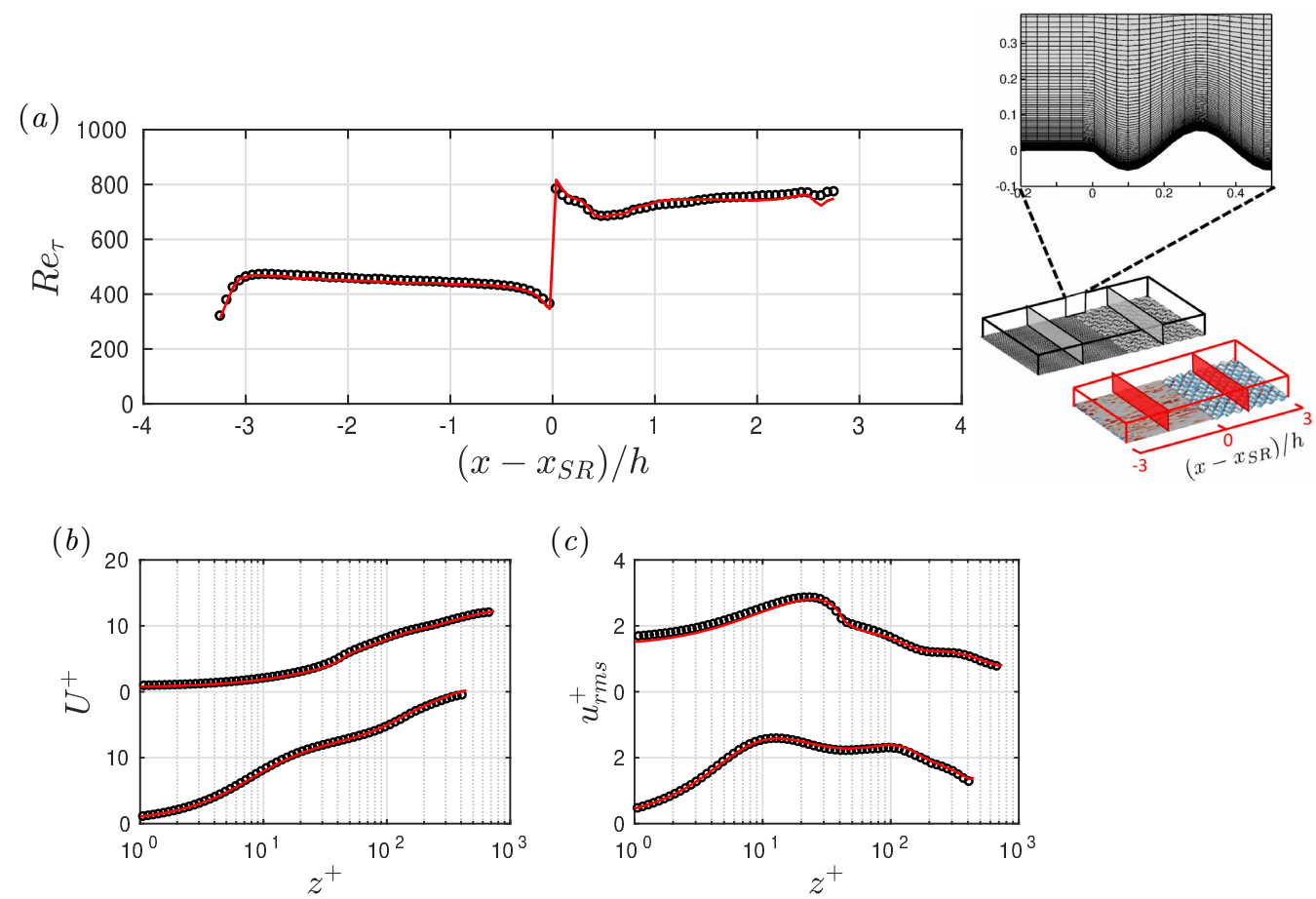

Figure 23: Comparison between cases $6 \mathrm{~h}(-)$ ) and 6h-verification (o). (a) $R e_{\tau}$ based on local $u_{\tau}$ and $h$. Profiles of $(b) U$ and $(c) u_{r m s}$. The quantities in plus units are normalised by the local $u_{\tau}$ and $\nu$. Comparison is made in the middle of the smooth patch (lower curves in $b, c$ ) and in the middle of the rough patch (upper curves in $b, c$ ), demonstrated in the domains on the right side.

conforming grid solver, from Cascade Technologies, Inc. (Ham et al. 2006), and is denoted as case 6 h-verification with the grid and domain size listed in table 6 . All the physical parameters are identical to case $6 \mathrm{~h}$ except the wall-normal grid (figure 23 on the right). For the body-conforming grid, the hyperbolic grid distribution starts from the bottom surface (as opposed to the roughness crest in case $6 \mathrm{~h}$ ). Despite the earlier grid stretching above the rough surface, $\Delta_{z}^{+}$(based on the local $u_{\tau}$ ) is maintained below unity up to the roughness crest. Figure 23 shows the comparison between cases $6 \mathrm{~h}$ and $6 \mathrm{~h}$-verification for the parameters of interest (including local $R e_{\tau}$, and profiles of $U$ and $u_{r m s}$ ), and good agreement (less than $3 \%$ difference) is obtained between the two cases.

With the chosen grid resolution in table 6 , one repeatable tile of "egg-carton" roughness with an area of $\lambda \times \lambda$ is resolved by $\lambda / \Delta_{x} \times \lambda / \Delta_{y} \simeq 25 \times 48=1200$ grid points in the $x y$-plane. Scotti (2006) who used IBM to implement the sand-grain roughness, resolved each roughness element by maximum 66 grid points in the $x y$-plane. Yuan \& Piomelli (2014) who also adopted Scotti's IBM and considered sand-grain roughness, resolved each roughness element by 16 grid points in the $x y$-plane.

\section{Appendix B. Periodic versus a rough-to-smooth non-periodic case}

In this appendix the periodic case $12 \mathrm{~h}$ (table 1 ) is compared with a non-periodic case with fully recovered inflow. Here, only the rough-to-smooth step change is considered due to the slow flow recovery over the smooth patch. For the non-periodic case, the concurrent 


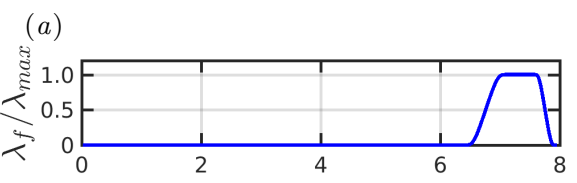

(b) $u^{\prime} / u_{\tau_{o}}$

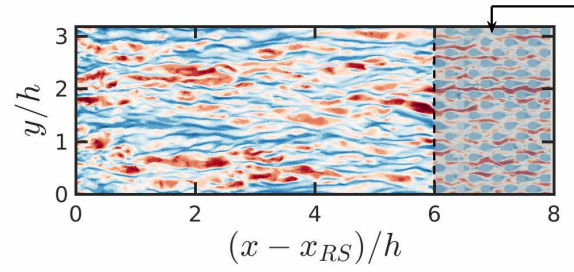

(c) $u_{p r e c}^{\prime} / u_{\tau_{o}}$

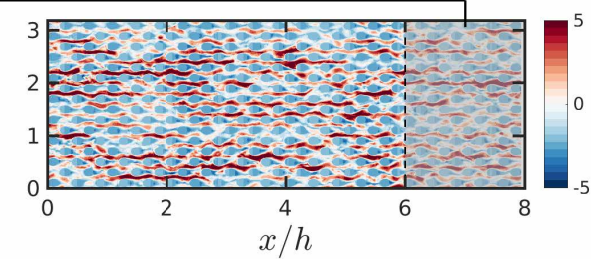

Figure 24: Illustration of concurrent precursor method (Stevens et al. 2014; Munters et al. 2016) for the rough-to-smooth non-periodic setup with fully recovered inflow; $(b)$ the main domain and $(c)$ the precursor domain at $z u_{\tau_{o}} / \nu=15$. The shaded regions indicate the data extraction region in $(c)$ and the fringe forcing region in $(b) .(a)$ the fringe masking function $\lambda_{f}$ normalised by $\lambda_{\max }=3000$.

$\begin{array}{cccccccc}\text { Case } & L_{x} / h & N_{x} \times N_{y} \times N_{z} & \Delta_{x_{s}}^{+}, \Delta_{x_{r}}^{+} & \Delta_{y_{s}}^{+}, \Delta_{y_{r}}^{+} & \left.\Delta_{z_{s}}^{+}\right|_{0},\left.\Delta_{z_{r}}^{+}\right|_{0} & \lambda / \Delta_{x} & \lambda / \Delta_{y} \\ \text { non-periodic } & 7.95 & 512 \times 384 \times 400 & 6.6,10.9 & 3.5,5.8 & 0.2,0.4 & 25.6 & 48.0\end{array}$

Table 7: Summary of the non-periodic case for the main simulation with $L_{y} / h=3.1808$. For the precursor simulation, the domain size and number of grid points is the same as the main simulation, and the resolution is the same as the main simulation over the rough (fringe) region.

precursor method (Stevens et al. 2014; Munters et al. 2016) was adopted to simulate a non-periodic flow with a periodic code (figure 24). This method consists of a precursor simulation, which here is a fully-recovered flow over homogeneous rough surface with a domain length of about $8 h$ (figure $24 c$ ), in addition to the main simulation, which here is a rough-to-smooth step change with a domain length of about $6 h$ smooth and $2 h$ rough (figure 24b). Both simulations are run synchronously with the same time steps, domain sizes and number of grid points in each direction. The precursor simulation is driven by a pressure gradient, which here is adjusted such that $R e_{\tau_{o}}=704$, the asymptotic Reynolds number downstream of the smooth-to-rough step change (figure $3 b$ ). The main simulation is driven by the imposed flow $\left(u_{\text {prec }, i}\right)$ from the precursor simulation (shaded area in figure 24c) through the fringe force $f_{f r, i}=-\lambda_{f}\left(u_{i}^{n}-u_{p r e c, i}^{n}\right)$, added to the right hand side of (A 1). The masking function $\lambda_{f}=\lambda_{\max }\left\{S_{f}\left[\left(x-x_{s}\right) / \Delta_{s}\right]-S_{f}\left[\left(x-x_{e}\right) / \Delta_{e}+1\right]\right\}$ (figure 24a), is non-zero only in the fringe region (shaded area in figure $24 b$ ). For $S_{f}$ the reader may refer to equation (4c) in Munters et al. (2016). With this forcing technique, the flow over the precursor homogeneous rough-wall simulation is copied to the end of the main simulation over its rough patch (consider the arrow from figure $24 c$ to figure $24 b$ ). The periodic boundary condition in the main simulation (figure $24 b$ ), recycles the fully developed flow over the rough patch to the beginning of the smooth patch, hence we simulate a rough-to-smooth step change with fully-developed oncoming flow over the rough surface.

The smooth patch length and resolution of the non-periodic case (table 7), are almost 
(a)

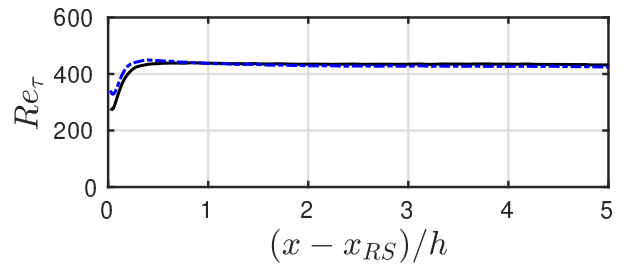

(b) $U^{+}$

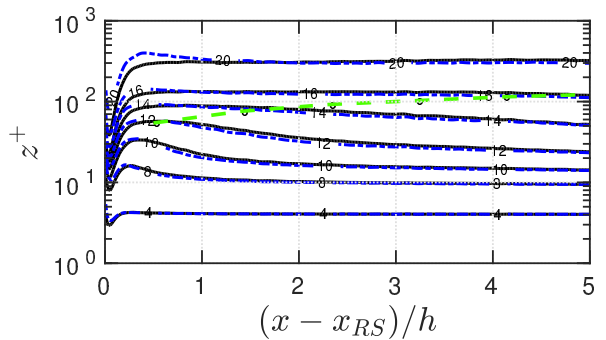

(c) $u_{r m s}^{+}$

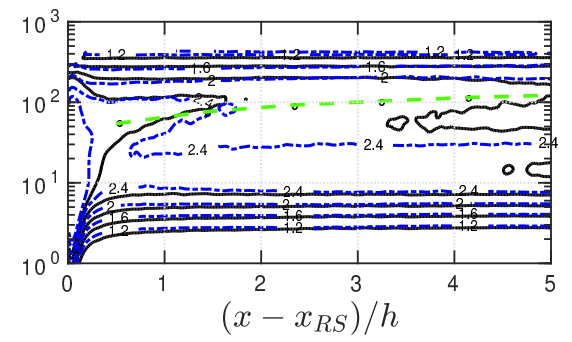

Figure 25: Comparison of statistics between case $12 \mathrm{~h}(-\cdot-)$ and non-periodic case () during rough-to-smooth step change. (a) $R e_{\tau}$; contour lines of (b) $U^{+}$and $(c) u_{r m s}^{+}$. Quantities in plus units are normalised by the local $u_{\tau}$ and $\nu$. The IBL thickness, defined by Elliott (1958), is overlaid on the contour lines (-- o-- )

identical to case $12 \mathrm{~h}$ (table 1$)$. A domain length of 20 roughness wavelengths $(20 \lambda \approx 8 h)$ is considered, which for the precursor simulation is homogeneously rough, and for the main simulation is partially smooth $(15 \lambda \approx 6 h)$ and partially rough $(5 \lambda \approx 2 h)$, in its fringe region. The input parameters for $\lambda_{f}$ are $\lambda_{\max }=3000, x_{s}=0.8 L_{x}, x_{e}=L_{x}, \Delta_{s}=0.1 L_{x}$ and $\Delta_{e}=0.05 L_{x}$; these parameters are adjusted according to Munters et al. (2016) to sufficiently damp the terms in (A 1) except $f_{f r, i}$, yet low enough for numerical stability.

Comparison of the statistics between the non-periodic case and the periodic case $12 \mathrm{~h}$ (figure 25) shows that the difference between these two cases in terms of $R e_{\tau}$ (figure $25 a$ ) is less than $1 \%$ after a fetch of $0.8 h$. The difference in terms of $U^{+}$and $u_{r m s}^{+}$(figure $25 b, c$ ) is less than $1 \%$ and $4 \%$, respectively, after a fetch of $0.3 h$ within the IBL $(--\circ--$, region of interest). The discrepancy up to a fetch of $0.8 h$ could be due to the forcing up to the very end of the fringe region (figure $24 a$ ). Nevertheless, the conclusions drawn from the analysis of $U^{+}$do not depend on this minor discrepancy: $\S 3.2$ on equilibrium assumption, and $\S 3.3$ on the suitable IBL definition. Also, $4 \%$ difference in $u_{r m s}^{+}$does not impact the conclusions drawn in $\S 3.2$. This appendix reinforces the domain length study in $\S 3.1$.

\section{REFERENCES}

Albertson, J. D. \& Parlange, M. B. 1999 a Natural integration of scalar fluxes from complex terrain. Adv. Water Resour. 23, 239-252.

Albertson, J. D. \& Parlange, M. B. $1999 b$ Surface length scales and shear stress: implications for land-atmosphere interaction over complex terrain. Water Resour. Res. 35, 2121-2132.

Anderson, W., Barros, J. M., Christensen, K. T. \& Awasthi, A. 2015 Numerical and experimental study of mechanisms responsible for turbulent secondary flows in boundary layer flows over spanwise heterogeneous roughness. J. Fluid Mech. 768, 316-347.

Andreopoulos, J. \& Wood, D. H. 1982 The response of a turbulent boundary layer to a short length of surface roughness. J. Fluid Mech. 118, 143-164. 
Antonia, R. A. \& Luxton, R. E. 1971 The response of a turbulent boundary layer to a step change in surface roughness part 1. smooth to rough. J. Fluid Mech. 48, 721-761.

Antonia, R. A. \& Luxton, R. E. 1972 The response of a turbulent boundary layer to a step change in surface roughness. part 2. rough-to-smooth. J. Fluid Mech. 53, 737-757.

Bou-Zeid, E., Meneveau, C. \& Parlange, M. B. 2004 Large-eddy simulation of neutral atmospheric boundary layer flow over heterogeneous surfaces: blending height and effective surface roughness. Water Resour. Res. 40, W02505.

Bou-Zeid, E., Meneveau, C. \& Parlange, M. B. 2005 A scale-dependent Lagrangian dynamic model for large eddy simulation of complex turbulent flows. Phys. Fluids 17, 025105.

Bou-Zeid, E., Parlange, M. B. \& Meneveau, C. 2007 On the parameterization of surface roughness at regional scales. J. Atmos. Sci. 64, 216-227.

Bradley, E. F. 1968 A micrometeorological study of velocity profiles and surface drag in the region modified by a change in surface roughness. Q. J. R. Meteorolog. Soc. 94, 361-379.

Brutsaert, W. 1998 Land-surface water vapor and sensible heat flux: spatial variability, homogeneity, and measurement scales. Water Resour. Res. 34, 2433-2442.

Calaf, M., Meneveau, C. \& Meyers, J. 2010 Large eddy simulation study of fully developed wind-turbine array boundary layers. Phys. Fluids 22, 015110.

CArper, M. A. \& Porté-Agel, F. 2008 Subfilter-scale fluxes over a surface roughness transition. part I: measured fluxes and energy transfer rates. Boundary Layer Meteorol. 126, 157-179.

Chamorro, L. P. \& Porté-Agel, F. 2009 Velocity and surface shear stress distributions behind a rough-to-smooth surface transition: a simple new model. Boundary Layer Meteorol. 130, 29-41.

Chan, L., MacDonald, M., Chung, D., Hutchins, N. \& Ooi, A. 2015 A systematic investigation of roughness height and wavelength in turbulent pipe flow in the transitionally rough regime. J. Fluid Mech. 771, 743-777.

Chan, L., MacDonald, M., Chung, D., Hutchins, N. \& Ooi, A. 2018 Secondary motion in turbulent pipe flow with three-dimensional roughness. J. Fluid Mech. 854, 5-33.

Cheng, H. \& Castro, I. P. 2002 Near-wall flow development after a step change in surface roughness. Boundary Layer Meteorol. 105, 411-432.

Chung, D., Chan, L., MacDonald, M., Hutchins, N. \& Ooi, A. 2015 A fast direct numerical simulation method for characterising hydraulic roughness. J. Fluid Mech. 773, 418-431.

Chung, D., Monty, J. P. \& OoI, A. 2014 An idealised assessment of townsends outer-layer similarity hypothesis for wall turbulence. J. Fluid Mech. 742, R3.

Dupont, S. \& BRunet, Y. 2009 Coherent structures in canopy edge flow: a large-eddy simulation study. J. Fluid Mech. 630, 93-128.

Efros, V. \& Krogstad, P. 2011 Development of a turbulent boundary layer after a step from smooth to rough surface. Exp. Fluids 51, 1563-1575.

Elliott, W. P. 1958 The growth of the atmospheric internal boundary layer. Trans. Am. Geophys. Union 39, 1048-1054.

Fadlun, E. A., Verzicco, R., Orlandi, P. \& Mohd-Yusof, J. 2000 Combined immersedboundary finite-difference methods for three-dimensional complex flow simulations. $J$. Comput. Phys. 161, 35-60.

Ham, F., Mattsson, K. \& Iaccarino, G. 2006 Accurate and stable finite volume operators for unstructured flow solvers. In Center of Turbulence Research Annual Research Breifs pp. 243-261. Stanford University.

Hanson, R. E. \& Ganapathisubramani, B. 2016 Development of turbulent boundary layers past a step change in wall roughness. J. Fluid Mech. 795, 494-523.

Ismail, U., ZaKi, T. A. \& Durbin, P. A. 2018 Simulations of rib-roughened rough-to-smooth turbulent channel flows. J. Fluid Mech. 843, 419-449.

JACOBI, I. \& McKeOn, B. J. 2011 New perspectives on the impulsive roughness-perturbation of a turbulent boundary layer. J. Fluid Mech. 677, 179-203.

Jegede, O. O. \& FoKen, T. H. 1999 A study of the internal boundary layer due to a roughness change in neutral conditions observed during the linex field campaigns. Theor. Appl. Climatol. 62, 31-41. 
Jiménez, J., Hoyas, S., Simens, M. P. \& Mizuno, Y. 2010 Turbulent boundary layers and channels at moderate Reynolds numbers. J. Fluid Mech. 657, 335-360.

Kaimal, J. C. \& Finnigan, J. J. 1994 Atmospheric boundary layer flows: their structure and measurement. Oxford University Press.

LEE, J. H. 2015 Turbulent boundary layer flow with a step change from smooth to rough surface. Int. J. Heat Fluid Flow 54, 39-54.

Lin, C. L. \& Glendening, J. W. 2002 Large eddy simulation of an inhomogeneous atmospheric boundary layer under neutral conditions. J. Atmos. Sci. 59, 2479-2497.

Loureiro, J. B. R., Sousa, F. B. C. C., Zotin, J. L. Z. \& Freire, A. P. S. 2010 The distribution of wall shear stress downstream of a change in roughness. Int. J. Heat Fluid Flow 31, 785-793.

Miller, N. E. \& Stoll, R. 2013 Surface heterogeneity effects on regional-scale fluxes in the stable boundary layer: aerodynamic roughness length transitions. Boundary Layer Meteorol. 149, 277-301.

MiYAKe, M. 1965 Transformation of the atmospheric boundary layer over inhomogeneous surfaces. Tech. Rep.. Washington University Department of Atmospheric Sciences.

Morinishi, Y., Lund, T. S., Vasilyev, O. V. \& Moin, P. 1998 Fully conservative higher order finite difference schemes for incompressible flow. J. Comput. Phys. 143, 90-124.

Mulhearn, P. J. 1978 A wind-tunnel boundary-layer study of the effects of a surface roughness change: rough to smooth. Boundary Layer Meteorol. 15, 3-30.

Munro, D. S. \& Oke, T. R. 1975 Aerodynamic boundary-layer adjustment over a crop in neutral stability. Boundary Layer Meteorol. 9, 53-61.

Munters, Wim, Meneveau, Charles \& Meyers, Johan 2016 Turbulent inflow precursor method with time-varying direction for large-eddy simulations and applications to wind farms. Boundary-layer meteorology 159 (2), 305-328.

Nickels, T. B. 2004 Inner scaling for wall-bounded flows subject to large pressure gradients. J. Fluid Mech. 521, 217-239.

Panofsky, H. A. \& Townsend, A. A. 1964 Change of terrain roughness and the wind profile. Q. J. R. Meteorolog. Soc. 90, 147-155.

Pendergrass, W. \& Arya, S. P. S. 1984 Dispersion in neutral boundary layer over a step change in surface roughness-I. mean flow and turbulence structure. Atmos. Environ. 18, $1267-1279$.

Perot, J. B. 1993 An analysis of the fractional step method. J. Comput. Phys. 108, 51-58.

Pope, S. B. 2000 Turbulent Flows. Cambridge University Press.

RAo, K. S., WyngaArd, J. C. \& Coté, O. R. 1974 The structure of the two-dimensional internal boundary layer over a sudden change of surface roughness. J. Atmos. Sci. 31, $738-746$.

Saito, N. \& Pullin, D. I. 2014 Large eddy simulation of smooth-rough-smooth transitions in turbulent channel flows. Int. J. Heat Mass Transfer 78, 707-720.

SAvelyev, S. A. \& TAYlor, P. A. 2005 Internal boundary layers: I. height formulae for neutral and diabatic flows. Boundary Layer Meteorol. 115, 1-25.

Scotti, A. 2006 Direct numerical simulation of turbulent channel flows with boundary roughened with virtual sandpaper. Phys. Fluids 18, 031701.

Silva-Lopes, A., Palma, J. M. L. M. \& Piomelli, U. 2015 On the determination of effective aerodynamic roughness of surfaces with vegetation patches. Boundary Layer Meteorol. 156, 113-130.

Spalart, P. R., Moser, R. D. \& Rogers, M. M. 1991 Spectral methods for the Navier-Stokes equations with one infinite and two periodic directions. J. Comput. Phys. 96, 297-324.

Stevens, Richard JAM, Graham, Jason \& Meneveau, Charles 2014 A concurrent precursor inflow method for large eddy simulations and applications to finite length wind farms. Renewable energy 68, 46-50.

Stoll, R. \& Porté-Agel, F. 2006 Dynamic subgrid-scale models for momentum and scalar fluxes in large-eddy simulations of neutrally stratified atmospheric boundary layers over heterogeneous terrain. Water Resour. Res. 42.

Verstappen, R. W. C. P. \& Veldman, A. E. P. 2003 Symmetry-preserving discretization of turbulent flow. J. Comput. Phys. 187, 343-368. 
Win, A. T., Mochizuki, S. \& Kameda, T. 2010 Response of the fully developed pipe flow to rough wall disturbances (mean velocity field). J. Fluid Sci. Tech. 5, 340-350.

Yang, X. I. A. \& Meneveau, C. 2017 Modelling turbulent boundary layer flow over fractal-like multiscale terrain using large-eddy simulations and analytical tools. Phil. Trans. R. Soc. A 375, 20160098.

Yuan, J. \& Piomelli, U. 2014 Numerical simulations of sink-flow boundary layers over rough surfaces. Phys. Fluids 26, 015113. 